\title{
Geochemical fingerprints and pebbles zircon geochronology: Implications for the provenance and tectonic setting of Lower Cretaceous sediments in the Zhucheng Basin (Jiaodong peninsula, North China)
}

\author{
Jin-Long Ni ${ }^{1,2}$, Jun-Lai Liu ${ }^{3, *}$, Xiao-Ling Tang ${ }^{4}$, Xiao-Xiao Shi ${ }^{3}$, Hong Zhang$^{1}$ and Shuai Han ${ }^{1}$ \\ ${ }^{1}$ Shandong Provincial Key Laboratory of Depositional Mineralization and Sedimentary Minerals, College of Earth \\ Sciences and Engineering, Shandong University of Science and Technology, Qingdao 266590, China. \\ ${ }^{2}$ Laboratory for Marine Mineral Resources, Qingdao National Laboratory for Marine Science and Technology, \\ Qingdao 266071, China. \\ ${ }^{3}$ State Key Laboratory of Geological Processes and Mineral Resources, China University of Geosciences, \\ Beijing 100083, China. \\ ${ }^{4}$ College of Chemistry 83 Environmental Engineering, Shandong University of Science and Technology, \\ Qingdao 266590, China. \\ *Corresponding author.e-mail: jliu@cubg.edu.cn
}

This paper conducts a petrogeochemical analysis of the Lower Cretaceous Laiyang Group's sandstones, compares the results with the Neoproterozoic and Mesozoic intrusive rocks in the southern Sulu Orogen (also called the Jiaonan Orogen), and performs an LA-ICP-MS zircon geochronology analysis of the granitic gneisses in the conglomerates of the Laiyang Group and the intrusive rocks in the Jiaonan Orogen. The results show that the major element proportions of the Longwangzhuang Formation (LWZ Fm) and Qugezhuang Formation (QGZ Fm) of the Laiyang Group in the Zhucheng Basin are similar. The values of various indices for the LWZ Fm are similar to the average sandstone content of active continental margins, whereas, the values for the QGZ Fm are similar to those of continental island arcs. The comparison shows that the REE characteristics of the LWZ Fm and QGZ Fm of Laiyang Group are similar to those of the Neoproterozoic granitic gneisses in the Jiaonan Orogen but obviously different from those of the Early Cretaceous intrusive rocks. A tectonic setting discrimination diagram reveals that the provenance of the Laiyang Group includes features of active continental margins and continental island arcs. A number of indicators, e.g., the sandstone type, the Chemical Index of Alteration, the Chemical Index of Weathering, the Plagioclase Index of Alteration and the Index of Chemical Constituent Variation, indicated that the sandstones did not undergo intense weathering and were deposited near the source area. The zircon ages of the granitic gneiss material in the conglomerates at the base of the Laiyang Group are $790 \pm 8.4 \mathrm{Ma}$, close to the ages of the Neoproterozoic granitic gneiss in the Jiaonan Orogen (739-819 Ma), and very different from the ages of the Early Cretaceous intrusive rocks. Combining with paleocurrent directions, geochemical character, the Neoproterozoic granitic gneisses in the Jiaonan Orogen may represent the primary provenance of the Laiyang Group in the Zhucheng Basin. During the depositional period of the Laiyang Group, the source rocks did not experience intense weathering, which indicated the Jiaonan Orogen experienced rapid uplift during this time, and coincided with the high exhumed rate of $2.0 \mathrm{~km} \mathrm{Ma}^{-1}$ from before ca. 128 to $123 \mathrm{Ma}$ in Jiaonan Orogen. The rapid subsidence during the formation of the Laiyang Group in the Zhucheng Basin and the rapid uplift of the Jiaonan Orogen are the result of a single regional extensional event associated with the lithospheric thinning and destruction of North China and peripheral cratons.

Keywords. Jiaodong peninsula; Sulu Orogen; Zhucheng Basin; Early Cretaceous; sandstones; petrogeochemistry.

J. Earth Syst. Sci., DOI 10.1007/s12040-016-0739-1, 125, No. 7, October 2016, pp. 1413-1437

(C) Indian Academy of Sciences 


\section{Introduction}

The North China Craton experienced a series of plate collision-suture activities between the late Paleozoic and the early-middle Mesozoic (Xiao et al. 2003; Suo et al. 2012). As the South China Block (SCB) subducted underneath the North China Craton and collided with it, the QinlingDabie-Sulu Orogen was formed in southern North China (Xu et al. 2009; Wang et al. 2012) (figure 1). Following this collision-suture process along its southern boundary, the North China Craton experienced intra-continental compressional deformation in the late Jurassic followed by extension (Ren et al. 2002), forming extensive extensional structures, such as rifted basins (Billia et al. 2013) and metamorphic core complexes (Liu et al. 2005, 2013) (figure 1).

Basins and orogens have constituted two basic tectonic units at the surface of the North China Craton since the late Mesozoic (Erikson et al. 2012; Luo et al. 2012; Holt et al. 2015). Researchers have primarily studied the basins that developed in the front edge of the orogeny, such as the Hefei Basin and Dabie Orogen, to identify the provenance characteristics and evolutionary history (Grimmer et al. 2003; Wang et al. 2003; Meng et al. 2007).

The Sulu Orogen, usually viewed as the eastern extension of the Dabie Orogen (Li et al. 2002, 2009, 2012), is developed on the eastern side of the famous Tan-lu Fault (figure 1). The Cretaceous Jiaolai Basin developed along the Sulu Orogen's northern margin (Zhang et al. 2003) provided a comprehensive window to understand the uplift and evolution processes of the Sulu Orogen. The Cretaceous Zhucheng Basin is located on the southern margin of the Jiaolai Basin and is adjacent to the Jiaonan Orogen (also referred to as the southern Sulu Orogen) (figure 2a). The Jiaonan Orogen is famous for its abundant eclogites (Chen et al. 2010; Wang et al. 2014), but the uplift rate of this orogen since the late Mesozoic is unknown. Additionally, few researchers have studied the relationship between this orogen and the Zhucheng Basin.

Many discoveries have been made by utilizing petrogeochemical analyses of the sandstones that developed in the frontal basins of orogens to determine the tectonic settings of the sandstone's formation, the provenance of the basins and the provenance tectonic settings (Adeigbe and Jimoh 2013; Billia et al. 2013; Ejeh et al. 2013; Ikhane et al. 2014). These studies provide another way to understand the features and evolution of basins and provenances. This paper focuses on samples from the Lower Cretaceous Laiyang Group of Zhucheng Basin, Jiaodong peninsula for sandstone petrogeochemical research to probe the source region's properties and paleo-weathering characteristics. This research is combined with a study of the zircon geochronology of the sandstones to uncover the coupled relationship between the uplift of the Jiaonan Orogen and the subsidence of the Zhucheng Basin.

\section{Geological setting}

The Jiaodong peninsula lies along the south-eastern margin of the North China Craton (Charles et al. 2011a), to the east of Tan-lu Fault. The area includes three tectonic units: the Jiaobei Uplift, the Sulu Orogen and an Early Cretaceous extensional basin (Charles et al. 2011b; Tang et al. 2014; Wu et al. 2014) (figure 2a).

The Jiaobei Uplift consists primarily of metasedimentary rocks from the Archean basement (Jiaodong Group and Jingshan Group), Paleoproterozoic Fenzishan Group and Neoproterozoic Penglai Group (Webb et al. 1999; Zhang 2012) and upper Mesozoic unmetamorphosed magmatic rocks (Goss et al. 2010; Charles et al. 2011a; Ma et al. 2014) associated with the Linglong Metamorphic Core Complex (MCC) (Charles et al. 2011b).

The Sulu Orogen is primarily composed of Neoproterozoic felsic gneiss and granitic gneisses (739-819 Ma) (Hacker et al. 2006; Huang et al. 2006), Paleoproterozoic meta-sedimentary rocks (1.9-2.1 Ga) (Xie et al. 2015) (e.g., the Jingshan Group and Fenzishan Group), which experienced ultrahigh-pressure metamorphism (Hacker et al. 2006), and unmetamorphosed Mesozoic plutons (Guo et al. 2005; Yang et al. 2005) associated with the Wulian MCC, which developed along the Sulu Orogen's southern segment (the Jiaonan Orogen) (Ni et al. 2013). The vast majority of the outcropping Mesozoic plutons are Lower Cretaceous intrusive rocks (Yang et al. 2005) (figure 2a).

The late Mesozoic extensional basin, called the Jiaolai Basin by previous researchers, is primarily filled with Lower Cretaceous Laiyang Group sedimentary rocks (135-120 Ma), Qingshan Group volcanic rocks (120-105 Ma) and the Upper Cretaceous Wangshi Group (85-65 Ma) (Zhang et al. 2003; Li et al. 2008) (figure 2b).

The Zhucheng Basin is located on the southern edge of the Jiaolai Basin, with a NE-SW-oriented depocenter parallel to the Sulu Orogen, and the exposed basin's basement consists primarily of Neoproterozoic granitic gneiss (figure 2c). The Laiyang Group is universally present in the Zhucheng Basin and has a sedimentary contact relationship with the Neoproterozoic granitic basement and a maximum thickness of $6000 \mathrm{~m}$. The Qingshan Group is a set of volcanic sedimentary rocks with uneven thicknesses and a maximum thickness of $3000 \mathrm{~m}$. The Wangshi Group is locally distributed and is relatively thin (figure 2c). 


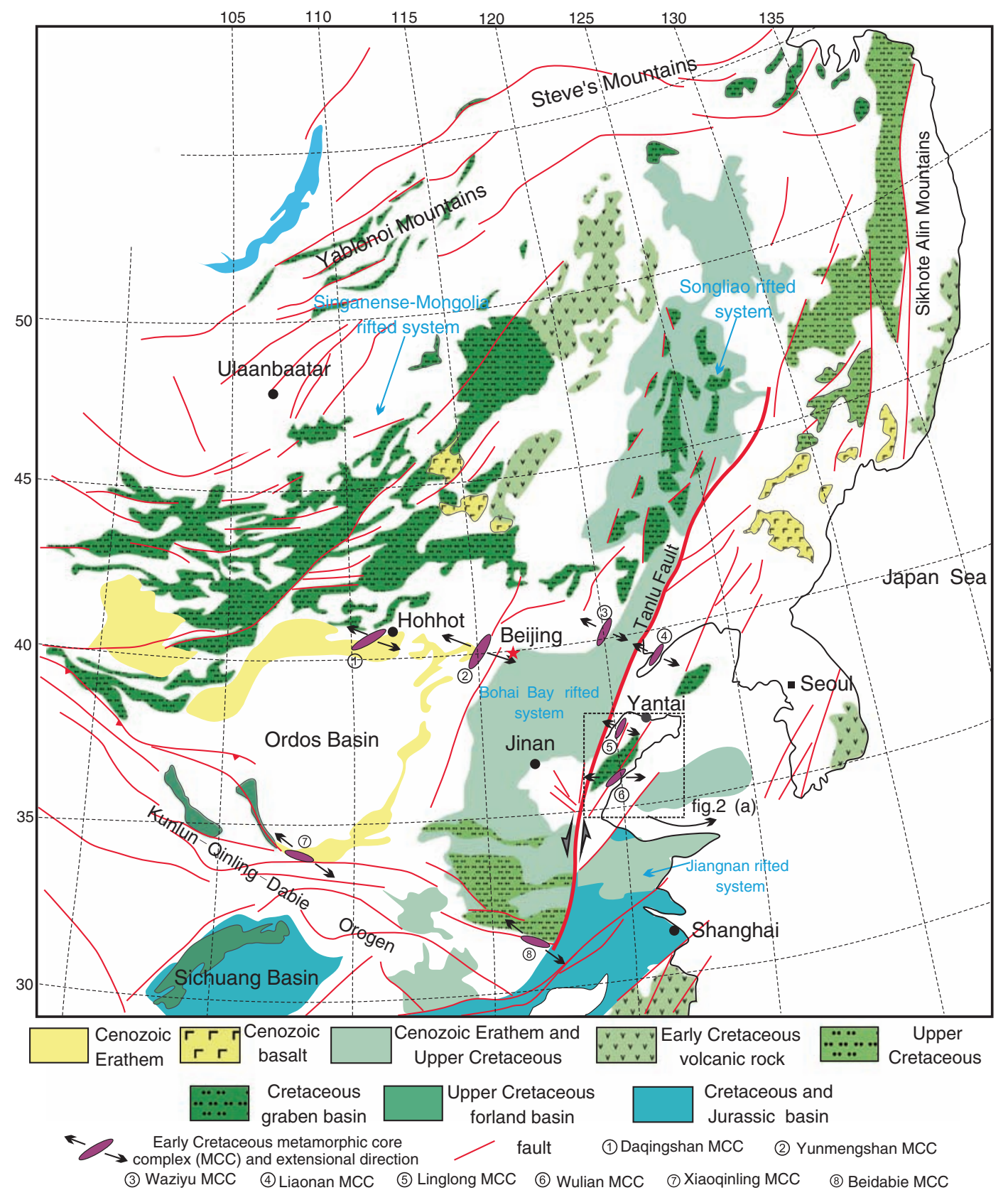

Figure 1. Late Mesozoic and Cenozoic extensional basin and tectonic framework of North China and its adjacent areas.

The Laiyang Group is a set of fluvial-lacustrine facies deposits with widespread parallel bedding, graded bedding (figure 3a, b), rhythmic bedding (figure 3c) and trough cross-bedding (figure 3d). The rock types primarily consist of grey sandstones, secondary shales, conglomerates and locally interbedded volcanic rocks. The Laiyang Group can be divided into the Linshansi Formation (kll), Longwangzhuang Formation (LWZ Fm; kllw), Qugezhuang Formation (QGZ Fm; klq) and Fajiaying Formation $(\mathrm{kl} f)$ from bottom to top (figure $2 \mathrm{~d}$ ). Based on geochronological data from the amphiboles and zircons in the basalts that intruded into the Laiyang Group and fossil data, the formation age of the Laiyang Group is approximately 135$120 \mathrm{Ma}$ (Zhang et al. 2003). The Qingshan Group, in which the Bamudi Formation (kqb) primarily developed, is a set of andesitic volcanic rocks, which lies on an angular unconformity over the Laiyang Group (figure 2d).

\section{Sample collection and analytical methods}

\subsection{Sample collection and paleocurrent directions}

Geological profiles with relatively good outcrops were chosen along field trip routes. Sandstones 

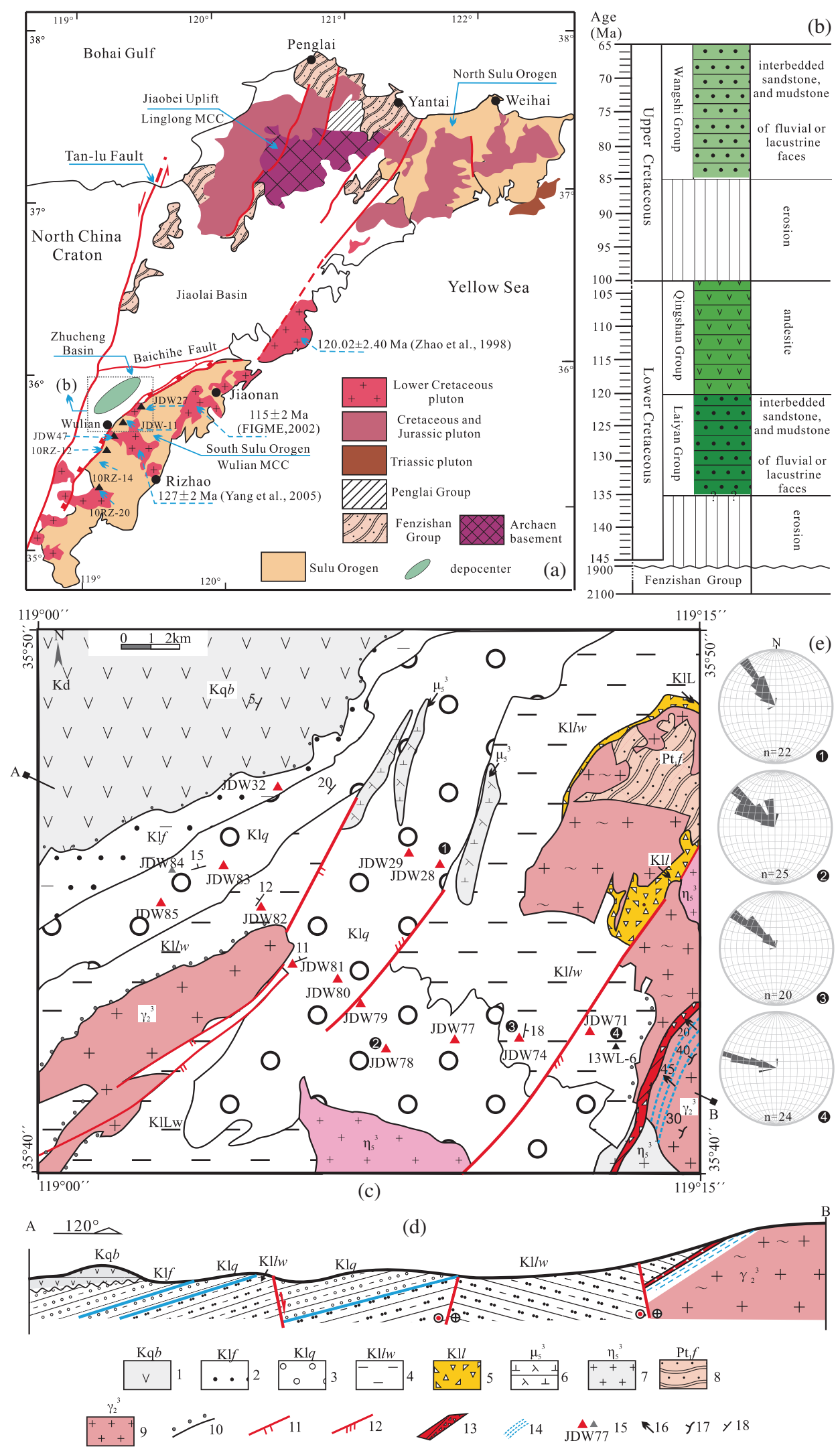

Figure 2. (a) Tectonic location map, (b) stratigraphic framework of the Cretaceous system, (c) simplified geologic map, (d) profile map of the Zhucheng Basin, and (e) paleocurrent directions rose diagrams. 1: Bamodi Formation in the Qingshan Group (Kqb); 2-5: Laiyang Group (K1L), 2: Fajiaying Formation (Klf), 3: QGZ Fm (Klq), 4: LWZ Fm (Kllw), 5: Linshansi Formation (Kll); 6: dioriticporphyrite; 7: monzonite; 8: Fenzishan Group; 9: Neoproterozoic granitic gneiss; 10: angular unconformity; 11: normal fault; 12: compressive shear fault; 13: extensional crushed zone; 14: detachment fault zone; 15: locations of samples or photographs; 16: stretching lineation; 17: gneissosity attitude; and 18: sedimentary rock attitude. 

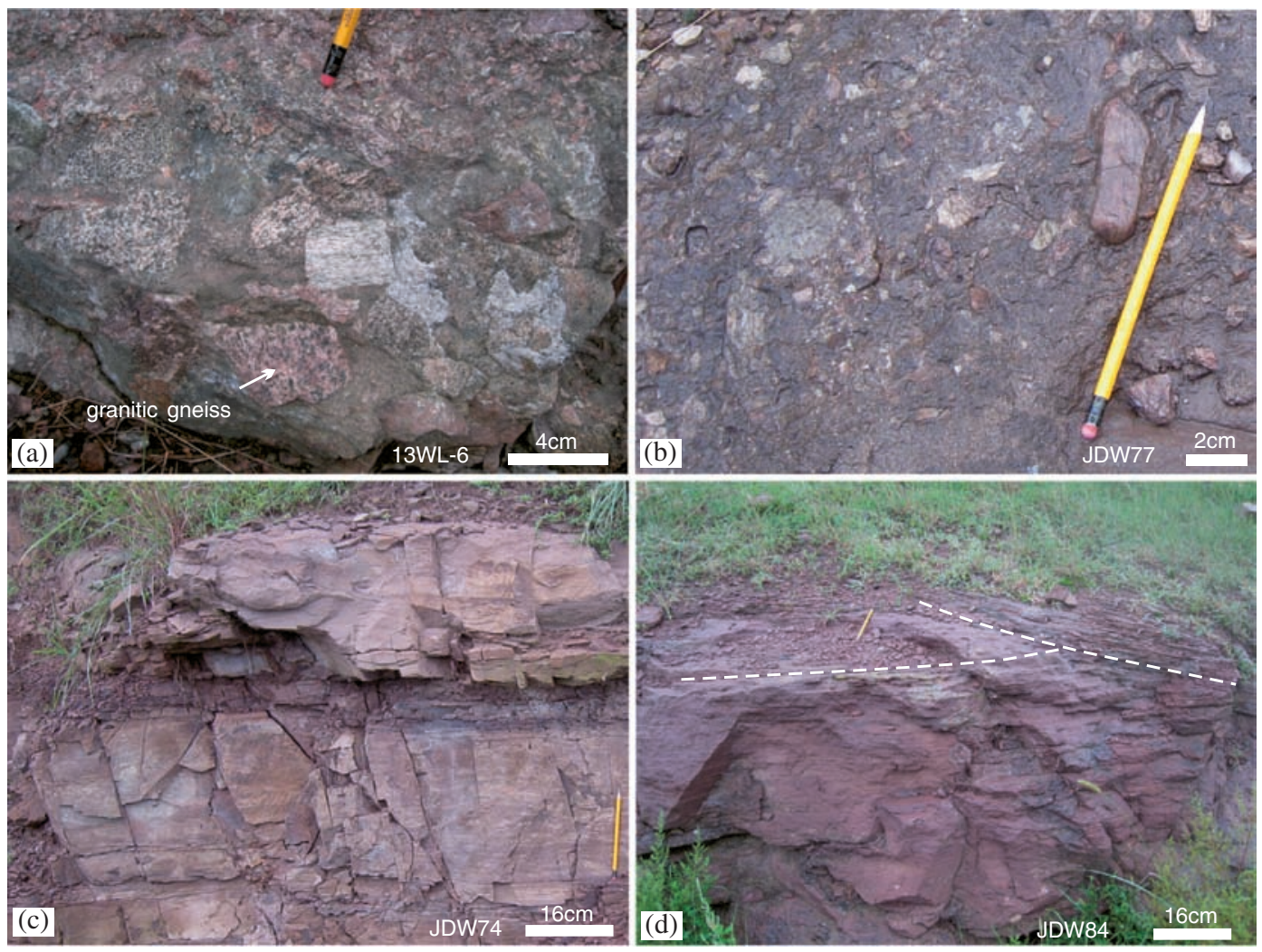

Figure 3. Photographs of the Laiyang Group's sedimentary rocks. (a) Conglomerate layer and a single-graded bed; (b) conglomeratic sandstone; (c) fine-grained sandstones interbedded with siltstone; (d) medium-grained sandstone with trough cross-bedding. The sample locations are shown in figure 2.

from close to the basin's basement westward to the Qingshan Group's volcanic rocks were collected for petrogeochemical analysis. The sampling sites were equally distributed (figure 2c).

Based on the collective features of the Laiyang Group, the sandstone in the Laiyang Group accounts for approximately $81-90 \%$ of the total thickness. The sizes of the grains influence the geochemical features of the sedimentary rocks (Pettijohn et al. 1972; Bhatia 1983); thus, the selection of sandstone samples for geochemical analysis should be based on the observation of specimens and thin sections. We collected 12 evenly mediumand fine-grained sandstones as analytical and test subjects. For comparison, we chose a small number of Neoproterozoic plutons and Mesozoic intrusions for the petrogeochemical analysis.

Conglomerate samples for zircon analysis were collected at the junction of the basin and orogen (figures $2 \mathrm{c}$ and $3 \mathrm{a}$ ). We selected gravel samples (clast diameters $>4 \mathrm{~cm}$ ) from conglomerates with clasts composed of granitic gneiss and then selected zircon grains from granitic gneiss. For comparison, we chose Neoproterozoic granitic gneiss and Mesozoic intrusive rocks for the zircon geochronology tests (figure 2a).

According to the occurrence of sandstone surface and oblique bedding or pebble dip, we drew the rose diagram of paleocurrent direction (figure 2e). Generally, the paleocurrent direction in Laiyang Group is NW-trending, indicating that the provenance is in the SE direction of the basin.

\subsection{Analytical methods}

Fresh whole-rock samples (13WL-6, JDW-11, 10RZ-14, 10RZ-20) were crushed in a steel crusher and powdered to grain size $<200$-mesh using an agate mill for major and trace-elemental analyses. Zircon grains were separated from heavy minerals by hand-picking under a binocular microscope. After zircons grains were mounted in epoxy, the resin discs were polished until the exposure of the grain centre of most zircon grains. Internal structures of zircon grains were imaged using the cathodeluminescence (CL). The CL images were obtained using a microprobe JEOL JXA-8900RL at the Institute of Geology and Geophysics, Chinese Academy of Sciences (CAS), Beijing (figure 4). The specific procedures are described in Xie et al. (2003).

\subsubsection{Major and trace element analysis}

The major elements of the sandstones were measured with a Regaku 3080E1 XRF spectrometer at 

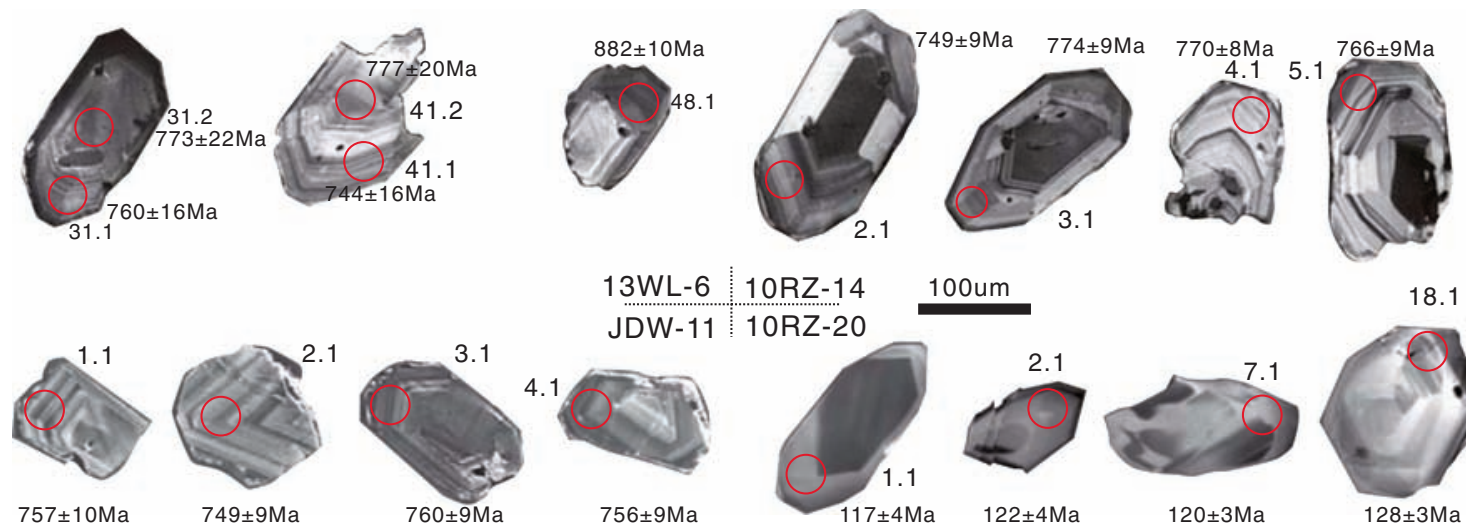

18.1

Figure 4. CL images of some dated zircons (with analytical sites marked, together with site numbers as listed in tables 3 and 4$)$.

the State Key Laboratory of Geological Processes and Mineral Resources, China University of Geosciences in Beijing. Analytical procedures are described in detail by Gao et al. (1995). The results are listed in table 1.

Trace element contents were analysed by double focusing inductively coupled plasma mass spectrometer (ICP-MS) at the Institute of Geology and Geophysics, Chinese Academy of Sciences (CAS), Beijing, using Finnigan MAT Element. Analytical procedures were described in detail by $\mathrm{Xu}$ et al. (2007). Analytical errors are generally better than $5 \%(2 \sigma)$. The analytical results are shown in table 2.

When processing the major element data, the loss on ignition was removed to calculate the percentages of each major element. The samples with $\mathrm{CaO}$ contents greater than $5 \%$ required special treatment because of the obvious calcite cementation. Generally, the highest $\mathrm{CaO}$ content to $\mathrm{Na}_{2} \mathrm{O}$ content ratio is $1: 1$ in silicates; the data were normalised according to this principle. After processing, the data approximate the real major element contents of the sandstones (table 1).

\subsubsection{Zircon morphology}

Four samples of the granitic gneiss conglomerate (13Wl-6), granitic gneiss (10RZ-14 and JDW-11), and syenite (10RZ-20) were used in this study. Typical CL images of the zircons are presented in figure 4, together with $\mathrm{U}-\mathrm{Pb}$ ages for relevant spots.

The zircons of samples 10RZ-14 (Th/U $=0.94-$ $1.67)$ and JDW-11 (Th/U=0.30-1.21) show magmatic oscillatory zones (figure $4 \mathrm{~b}, \mathrm{c}$ ). The zircons of sample 13WL-6 ( $\mathrm{Th} / \mathrm{U}=0.77-2.15)$ have two kinds of oscillatory zones; one kind of magmatic oscillatory zone is obvious, i.e., $13 \mathrm{WL}-6-31,13 \mathrm{WL}-$ 6-41 (figure 4a), being similar to those of the former two samples, the other kind of oscillatory zones are weak, e.g., 13WL-6-48.1 (figure 4a). The zircons of sample 10RZ-20 (Th/U=1.29-2.02) are different from the zircons of the former three samples, although they show the zones, they have unobvious oscillatory zones.

\subsection{3 $\mathrm{U}-\mathrm{Pb}$ dating}

U-Pb dating of zircons (10RZ-14) was conducted synchronously by the LA-ICP-MS at the State Key Laboratory of Geological Processes and Mineral Resources, China University of Geosciences, Wuhan. 13WL-6, JDW-11 and 10RZ-20 were conducted synchronously by the LA-ICP-MS at the State Key Laboratory of Geological Processes and Mineral Resources, China University of Geosciences, Beijing. Laser sampling was performed using a GeoLas 2005. An Agilent 7500a ICP-MS instrument was used to acquire ion-signal intensities. The specific methods are described in Wiedenbeck (1995), Andersen (2002) and Jackson et al. (2004). The ages of the granitic gneisses are shown as the weighted mean ages of ${ }^{206} \mathrm{~Pb} /{ }^{238} \mathrm{U}$, with $1 \sigma$ errors and a 95\% confidence interval for the weighted mean age. The test and processing results are shown in tables 3 and 4 and figure 5 .

\section{Results}

\subsection{Zircon chronology}

\subsubsection{Granitic gneiss in the conglomerates}

The gravels in the conglomerate have complex and various components, containing quartzites, marbles, granitic gneisses, etc. We choose the zircons from four granitic gneiss pebble samples, whose diameters are $7-10 \mathrm{~cm}$.

Forty-eight points were analysed among the selected zircons from the granitic gneisses in the conglomerates (13WL-6), and eight points were rejected due to low precisions (table 3 , figure $5 \mathrm{a}$ ). 
Table 1. Major oxide results for the sandstones in the Laiyang Group from the Zhucheng Basin and the Proterozoic and Mesozoic intrusive rocks in the Jiaonan Orogen.

\begin{tabular}{|c|c|c|c|c|c|c|c|c|c|c|c|c|c|c|c|c|}
\hline Sample source & Rock no. & $\mathrm{SiO}_{2}$ & $\mathrm{TiO}_{2}$ & $\mathrm{Al}_{2} \mathrm{O}_{3}$ & $\mathrm{Fe}_{2} \mathrm{O}_{3}^{\mathrm{T}}$ & $\mathrm{MnO}$ & $\mathrm{MgO}$ & $\mathrm{CaO}$ & $\mathrm{CaO}^{*}$ & $\mathrm{Na}_{2} \mathrm{O}$ & $\mathrm{K}_{2} \mathrm{O}$ & $\mathrm{P}_{2} \mathrm{O}_{5}$ & Tot & $\mathrm{K} / \mathrm{N}$ & FM & $\mathrm{S} / \mathrm{A}$ \\
\hline \multirow[t]{4}{*}{ LWZ Fm } & 71 & 73.67 & 0.44 & 12.48 & 2.52 & 0.04 & 1.03 & 3.1 & 3.21 & 4 . & 1.91 & 13 & 100 & .42 & 3.56 & 5.88 \\
\hline & JDW74 & 71.99 & 0.56 & 11.83 & 3.43 & 0.05 & 2.12 & 4.78 & 2.88 & 2.88 & 1.94 & 0.16 & 100 & 0.67 & 5.55 & 6.25 \\
\hline & JDW82 & 72.93 & 0.37 & 12.01 & 1.87 & 0.04 & 0.23 & 6.2 & 3.24 & 3.24 & 2.63 & 0.11 & 100 & 0.81 & 2.11 & 6.25 \\
\hline & Average & 72.86 & 0.46 & 12.11 & 2.61 & 0.04 & 1.13 & 4.69 & 3.11 & 3.56 & 2.16 & 0.13 & 100 & 0.63 & 3.74 & 6.13 \\
\hline \multirow[t]{10}{*}{ QGZ Fm } & 28 & 62.79 & 0.96 & 16.17 & 7.83 & 0.07 & 2.16 & 1.6 & 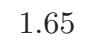 & 6.27 & 1.81 & .30 & 100 & 0.29 & 9.99 & 3.85 \\
\hline & JDW29 & 66.01 & 0.68 & 15.59 & 5.52 & 0.08 & 2.39 & 2.27 & 2.36 & 4.59 & 57 & 23 & 100 & 0.56 & 7.90 & 4.17 \\
\hline & JDW77 & 63.34 & 0.79 & 15.17 & 6.11 & 0.08 & 1.57 & 4.55 & 4.74 & 5.25 & 2.73 & .21 & 100 & 0.52 & 7.69 & 4.17 \\
\hline & JDW78 & 75.67 & 0.35 & 13.23 & 2.53 & 0.04 & 0.97 & 0.32 & 0.32 & 3.27 & 3.48 & 12 & 100 & 1.06 & 3.51 & 5.88 \\
\hline & JDW79 & 70.39 & 0.53 & 15.41 & 4.46 & 0.08 & 1.24 & 6.57 & 2.07 & 2.07 & 3.54 & .20 & 100 & 0.38 & 4.98 & 4.55 \\
\hline & JDW80 & 72.86 & 0.46 & 12.79 & 3.23 & 0.07 & 1.47 & 1.86 & 1.90 & 5.08 & 1.95 & 19 & 100 & 0.38 & 4.70 & 5.56 \\
\hline & JDW81 & 72.48 & 0.47 & 12.49 & 4.01 & 0.10 & 1.98 & 4.81 & 2.58 & 2.58 & 3.15 & 15 & 100 & 1.22 & 5.85 & 5.88 \\
\hline & JDW83 & 76.43 & 0.43 & 12.39 & 2.54 & 0.04 & 0.34 & 5.28 & 2.68 & 2.68 & 2.29 & 0.16 & 100 & 0.85 & 2.79 & 6.25 \\
\hline & JDV & 69.69 & 0.56 & 14.70 & 4.03 & 0.09 & 1.14 & 3.04 & 3.14 & 4.13 & 2.35 & 17 & 100 & 0.57 & 5.17 & 4.76 \\
\hline & Average & 69.96 & 0.58 & 14.22 & 4.47 & 0.07 & 1.47 & 3.37 & 2.38 & 3.99 & 2.65 & 0.19 & 100 & 0.65 & 5.84 & 5.26 \\
\hline \multirow{2}{*}{$\begin{array}{l}\text { Fajiaying Fm } \\
\text { Laiyang Group }\end{array}$} & & 88 & 0.63 & 20 & 4.74 & 0.03 & 1.29 & 1.85 & 1 & 7 & 4 & 18 & 100 & 0.42 & 5.87 & 4.53 \\
\hline & Av & 70.55 & 0.56 & 13.80 & 4.06 & 0.06 & 1.38 & 3.56 & 2.51 & 3.97 & 2.50 & .18 & 100 & 0.63 & 5.36 & 5.2 \\
\hline \multirow{4}{*}{$\begin{array}{l}\text { Neoproterozoic } \\
\text { granitic gneiss }\end{array}$} & 12 & 75.30 & 0.21 & 89 & 1.77 & 0.03 & 0.36 & 0.67 & & 0.00 & 4.59 & 07 & 100 & 1.29 & 2.13 & 5.84 \\
\hline & 10RZ14 & 77.26 & 0.17 & 12.47 & 0.89 & 0.05 & 0.10 & 0.35 & & 4.37 & 4.23 & 0.01 & 100 & 0.97 & 0.99 & 6.20 \\
\hline & JDW11 & 76.27 & 0.21 & 13.31 & 1.02 & 0.13 & 0.15 & 0.36 & & 6.34 & 1.90 & 0.04 & 100 & 1.03 & 1.47 & 6.15 \\
\hline & Average & 76.28 & 0.20 & 12.89 & 1.23 & 0.07 & 0.20 & 0.46 & & 76 & 3.57 & 0.04 & 100 & 1.10 & 1.53 & 6.06 \\
\hline \multirow{4}{*}{$\begin{array}{c}\text { Early Cretaceous } \\
\text { intrusive rocks }\end{array}$} & 10RZ20 & & 0.85 & & 7.50 & 0.09 & 2.68 & 3.19 & & & 1.00 & & 100 & 1.03 & 7.66 & 3.63 \\
\hline & JDW27 & 60.26 & 0.59 & 17.02 & 6.05 & 0.11 & 1.86 & 2.47 & & 4.39 & 3.82 & 0.35 & 100 & 0.87 & 7.91 & 3.54 \\
\hline & JDW47 & 62.12 & 0.58 & 15.20 & 4.60 & 0.13 & 2.06 & 2.86 & & 3.70 & 4.74 & 0.40 & 100 & 1.28 & 6.66 & 4.09 \\
\hline & Average & 60.88 & 0.67 & 16.28 & 5.21 & 0.11 & 2.20 & 3.04 & & 4.15 & 4.35 & 0.40 & 100 & 1.05 & 7.41 & 3.74 \\
\hline \multicolumn{2}{|l|}{ OIA } & & 1.00 & & 1.00 & 0.15 & 3.65 & 0.00 & & & .000 & 0.200 & & 0.39 & 0.0 & 3.44 \\
\hline \multicolumn{2}{|l|}{ CIA } & 70.69 & 0.64 & 14.04 & 4.82 & 0.1 & 1.97 & 2.68 & & 3.21 & 1.89 & 0.16 & & 0.59 & 6.79 & 5.03 \\
\hline \multicolumn{2}{|l|}{$\mathrm{ACM}$} & 73.86 & 0.46 & 12.89 & 3.06 & 0.1 & 1.23 & 2.48 & & 2.77 & 2.90 & 0.09 & & 1.05 & 4.29 & 5.73 \\
\hline \multicolumn{2}{|l|}{ PCM } & 81.95 & 0.49 & 8.41 & 3.28 & 0.05 & 1.39 & 1.89 & & 1.07 & 1.71 & 0.12 & & 1.60 & 4.67 & 9.74 \\
\hline \multicolumn{2}{|l|}{$\mathrm{UCC}$} & 66.6 & 0.64 & 15.4 & & 0.1 & 2.48 & 3.59 & & 3.27 & 2.80 & 0.15 & & 0.86 & & 4.32 \\
\hline
\end{tabular}

$\mathrm{S} / \mathrm{A}=\mathrm{SiO}_{2} / \mathrm{Al}_{2} \mathrm{O}_{3} ; \mathrm{K} / \mathrm{N}=\mathrm{K}_{2} \mathrm{O} / \mathrm{Na}_{2} \mathrm{O} ; \mathrm{FM}=\mathrm{Fe}_{2} \mathrm{O}_{3}^{\mathrm{T}}+\mathrm{MgO} ;$ OIA: ocean island average chemical composition; CIA: continental island arc sandstone average chemical composition; ACM: active continental margin sandstone average chemical composition; PCM: passive continental margin sandstone average chemical composition. The above data are derived from Bhatia (1983). UCC: upper continental crust average chemical composition, derived from Rudnick and Gao (2003).

The observed ${ }^{206} \mathrm{~Pb} /{ }^{238} \mathrm{U}$ zircon ages fall between $744 \pm 15$ and $882 \pm 26 \mathrm{Ma}$. Among these zircon ages, $744 \pm 15$ Ma might be the latest zircon crystallisation age of the granitic gneiss, and the zircon of $882 \pm 26$ Ma might be an older magmatic zircon, which represents the older crystallisation age (figure 4a-48.1). The weighted mean age is $790 \pm 8.4$ Ma (MSWD=3.1), which represents these granitic gneisses belong to Neoproterozoic.

\subsubsection{Felsic intrusions in the Jiaonan Orogen}

Three samples from the intrusions in the Jiaonan Orogen were chosen to conduct zircon geochronology tests, and all the analysed points were on the oscillatory zoned margins of the zircon grains.

Sample 10RZ-14 is granitic gneiss with 15 analyses, among which eight points had reliable precision. The acquired ${ }^{206} \mathrm{~Pb} /{ }^{238} \mathrm{U}$ zircon ages are relatively similar, between $764 \pm 11$ and $777 \pm 10 \mathrm{Ma}$, and have a ${ }^{206} \mathrm{~Pb} /{ }^{238} \mathrm{U}$ weighted mean age of $771 \pm 6.4 \mathrm{Ma}$ (MSWD $=0.21$ ) (table 4, figure 5b), indicating that this granitic gneiss was emplaced in the Neoproterozoic.

Sample JDW-11 is granitic gneiss that intruded into sample 10RZ-14 based on field observations. Nineteen points were analysed, of which all points met the required precision. The ${ }^{206} \mathrm{~Pb} /{ }^{238} \mathrm{U}$ ages are between $728 \pm 10$ and $786 \pm 10 \mathrm{Ma}$, with a weighted mean age of $751 \pm 6.3 \mathrm{Ma}(\mathrm{MSWD}=1.9)$ (table 4, figure 5c), indicating that this granitic gneiss was also emplaced in the Neoproterozoic.

Sample 10RZ-20 is syenite that was emplaced into Neoproterozoic granitic gneiss. Eighteen points were analysed, and 17 points met the required precision. The ages of the points are primarily between $120 \pm 3$ and $141 \pm 5 \mathrm{Ma}$, and the ${ }^{206} \mathrm{~Pb} /{ }^{238} \mathrm{U}$ weighted mean age is $126 \pm 3$ Ma $(\mathrm{MSWD}=2.9)$ (table 4 , figure 5d), indicating that the syenite intruded in the early Cretaceous. 


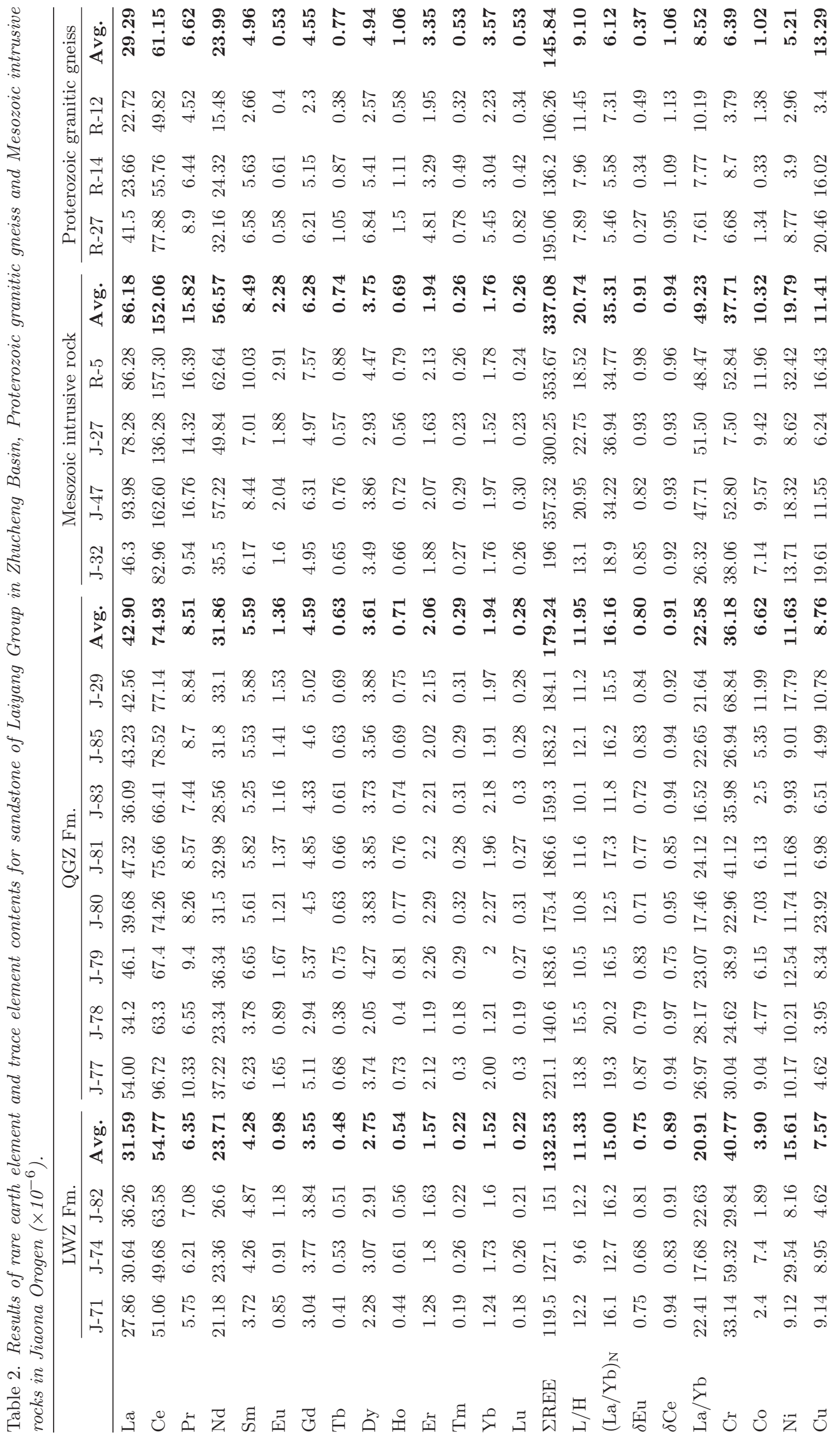




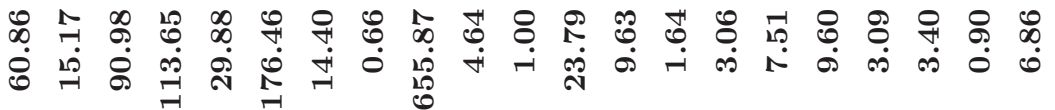
疋

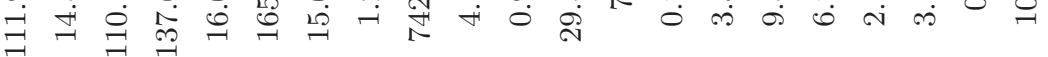

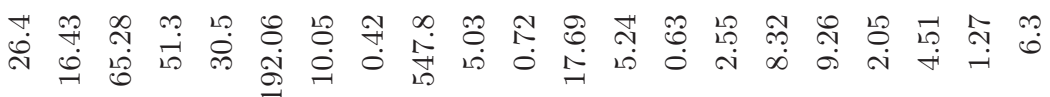

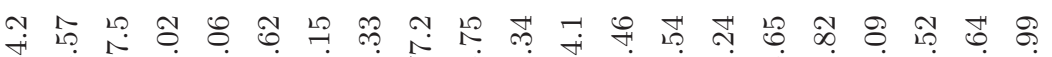
Ұ

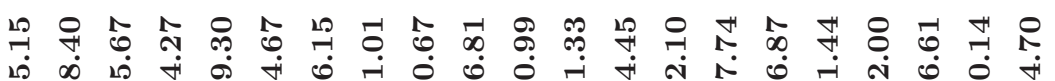

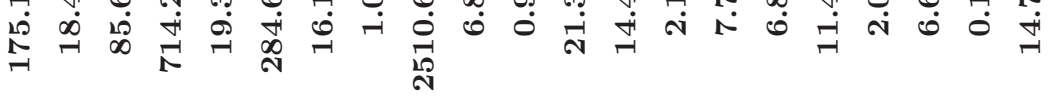
ๆ

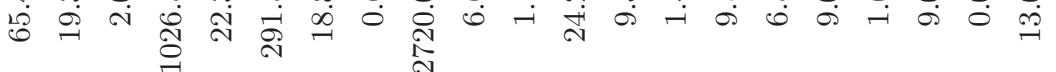
온

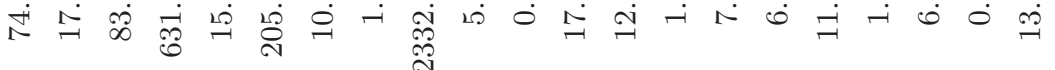

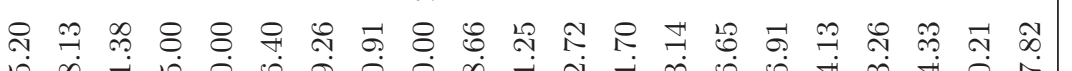

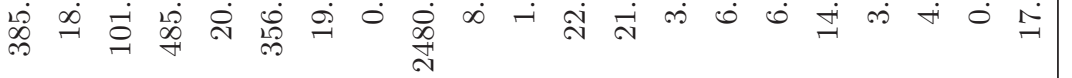

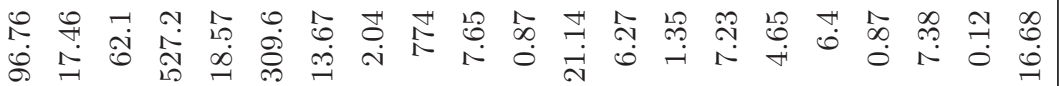
F

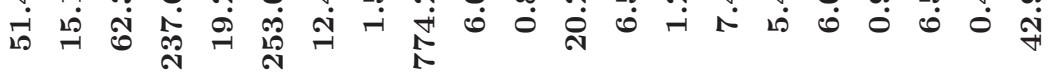
น0 ○ 跠

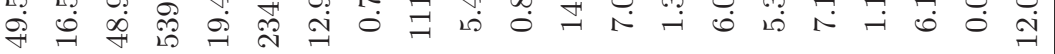

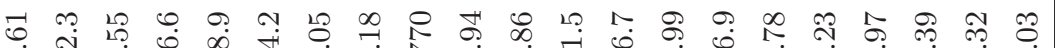
तิ 舟

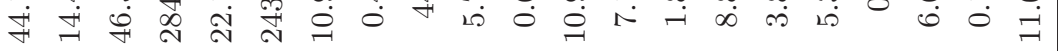
施

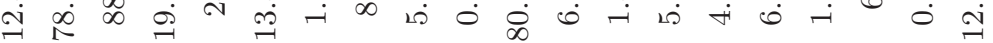

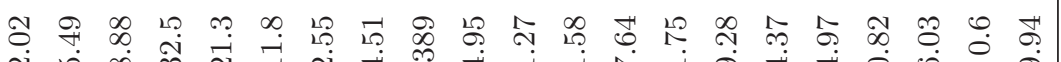

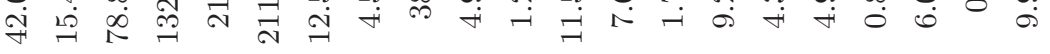

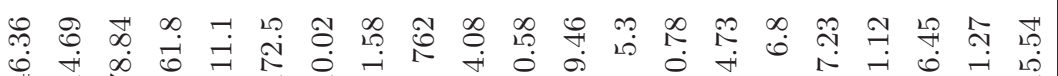

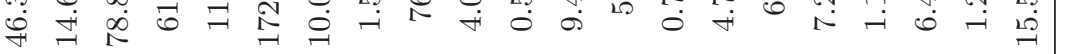
웅 ㄷำ

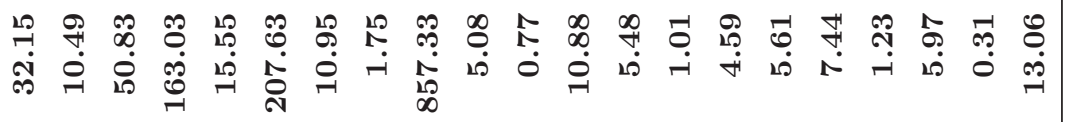
苞尔

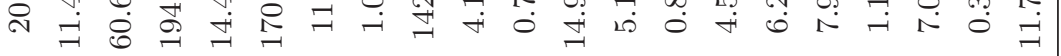

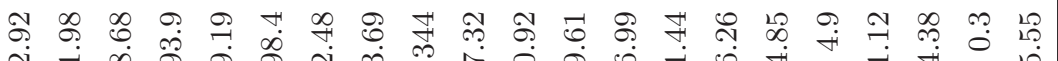

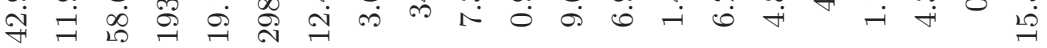
궁

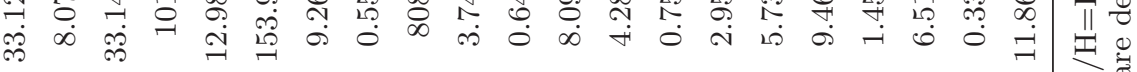
งี 
Table 3. LA-ICP-MS U-Pb zircon age data for the granitic gneiss conglomerates from the base of the Zhucheng Basin.

\begin{tabular}{|c|c|c|c|c|c|c|c|c|c|c|c|c|c|c|}
\hline \multirow[b]{2}{*}{ Spot } & \multirow[b]{2}{*}{$\begin{array}{c}\mathrm{U} \\
(\mathrm{ppm})\end{array}$} & \multirow[b]{2}{*}{$\begin{array}{c}\text { Th } \\
(\mathrm{ppm})\end{array}$} & \multirow[b]{2}{*}{$\mathrm{Th} / \mathrm{U}$} & \multirow[b]{2}{*}{$\begin{array}{c}\mathrm{Pb} \text { (total) } \\
(\mathrm{ppm})\end{array}$} & \multicolumn{6}{|c|}{ Isotopic ratios } & \multicolumn{4}{|c|}{ Age (Ma) } \\
\hline & & & & & $\begin{array}{c}{ }^{207} \mathrm{~Pb} / \\
{ }^{206} \mathrm{~Pb}\end{array}$ & $1 \sigma$ & $\begin{array}{c}{ }^{207} \mathrm{~Pb} / \\
{ }^{235} \mathrm{U}\end{array}$ & $1 \sigma$ & $\begin{array}{c}{ }^{206} \mathrm{~Pb} / \\
{ }^{238} \mathrm{U}\end{array}$ & $1 \sigma$ & $\begin{array}{c}{ }^{207} \mathrm{~Pb} / \\
{ }^{235} \mathrm{U}\end{array}$ & $1 \sigma$ & $\begin{array}{c}{ }^{206} \mathrm{~Pb} / \\
{ }^{238} \mathrm{U}\end{array}$ & 1 \\
\hline \multicolumn{15}{|c|}{ Sample 13WL-6 $\left(\mathrm{E} 119^{\circ} 13^{\prime} 42^{\prime \prime}, \mathrm{N} 35^{\circ} 44^{\prime} 57^{\prime \prime}\right),{ }^{206} \mathrm{~Pb} /{ }^{238} \mathrm{U}$ age: $790 \pm 8.4 \mathrm{Ma}, \mathrm{MSWD}=3.1$} \\
\hline 1.1 & 125.71 & 129.08 & 1.03 & 29.75 & 0.0656 & 0.0037 & 1.1462 & 0.0569 & 0.1276 & 0.0025 & 775 & 27 & 774 & 10 \\
\hline 2.1 & 235.78 & 330.29 & 1.40 & 63.39 & 0.0688 & 0.0035 & 1.2076 & 0.0643 & 0.1241 & 0.0018 & 804 & 30 & 754 & 11 \\
\hline 3.1 & 149.27 & 196.91 & 1.32 & 44.94 & 0.0703 & 0.0037 & 1.3347 & 0.0694 & 0.1344 & 0.0023 & 861 & 30 & 813 & 13 \\
\hline 4.1 & 360.52 & 773.90 & 2.15 & 147.46 & 0.0684 & 0.0030 & 1.2643 & 0.0525 & 0.1323 & 0.0022 & 830 & 24 & 801 & 10 \\
\hline 5.1 & 181.99 & 140.80 & 0.77 & 38.12 & 0.0645 & 0.0033 & 1.1515 & 0.0567 & 0.1275 & 0.0023 & 778 & 27 & 773 & 13 \\
\hline 6.1 & 116.97 & 129.96 & 1.11 & 29.47 & 0.0643 & 0.0040 & 1.1524 & 0.0688 & 0.1280 & 0.0025 & 778 & 32 & 777 & 14 \\
\hline 7.1 & 97.79 & 93.58 & 0.96 & 23.12 & 0.0689 & 0.0050 & 1.2472 & 0.0800 & 0.1332 & 0.0029 & 822 & 36 & 806 & 17 \\
\hline 8.1 & 136.50 & 142.79 & 1.05 & .28 & 0.0648 & 0.0037 & 1.1556 & 0.0677 & 0.1263 & 0.0024 & 780 & 32 & 767 & 14 \\
\hline 9.1 & 146.39 & 142.04 & 0.97 & 17 & 0.0667 & 0.0037 & 1.1943 & 0.0638 & 0.1281 & 0.0027 & 798 & 30 & 777 & 15 \\
\hline 10.1 & 123.59 & 123.98 & 1.00 & 66 & 0.0673 & 0.0041 & 1.1785 & 0.0702 & & & 791 & 33 & 768 & \\
\hline 11.1 & 127.85 & 113.20 & 0.89 & 28.40 & 0.0621 & 0.0042 & 1.1524 & 0.0745 & 0.1333 & 0.0026 & 778 & 35 & 807 & 15 \\
\hline 12.1 & 153.54 & 161.73 & 1.05 & & 0.0689 & 0.0036 & 1.2399 & 0.0618 & 0.1304 & & 9 & 28 & 790 & 14 \\
\hline 15.1 & 107.12 & 105.71 & 0.99 & 2 & 0.0684 & 0.0041 & 1.2290 & 0.0682 & & & 814 & 31 & 792 & \\
\hline 17.1 & 96.12 & 87.54 & 0.91 & 20 & 0.0662 & 0.0038 & 1.2358 & 0.0738 & 0.1341 & 0.00 & 817 & 3 & 811 & 15 \\
\hline 20.1 & 151.39 & 156.94 & 1.04 & 04 & 0.0658 & 0.0035 & 1.1603 & 0.0637 & 0.1276 & 0. & 782 & 30 & 774 & 13 \\
\hline 21.1 & 87.77 & 83.06 & 0.95 & 7 & 0.0659 & 0.0049 & 1.1461 & 0.0729 & 0.1281 & 0.0 & 775 & 34 & 777 & 16 \\
\hline 23.1 & 338.94 & 307.82 & 0.91 & 80.89 & 0.0700 & 0.0027 & 1.3342 & 0.0499 & 0.1380 & 0.0 & 861 & 22 & 833 & 11 \\
\hline 24.1 & 59.42 & 82.64 & 1.39 & & 0.0724 & 0.0075 & 1.3248 & 0.1266 & 326 & 6 & 7 & 55 & 803 & 20 \\
\hline 25.1 & 107.21 & 106.59 & 0.99 & 24.41 & 0.0632 & 0.0043 & 1.1213 & 0.0745 & 0.1293 & 0.0025 & 764 & 36 & 784 & 14 \\
\hline 26.1 & 139.86 & 139.48 & 1.00 & 31.88 & 0.0657 & 0.0034 & 1.1652 & 0.0613 & 0.1285 & 0.0025 & 784 & 29 & 780 & 14 \\
\hline 27.1 & 130.08 & & & & & & & & & & & 31 & 797 & 1 \\
\hline 28.1 & 97.70 & 90.56 & 0.93 & 0 & 0.0638 & 0.0041 & 1.1363 & 0.0743 & 0.1278 & 0.00 & 771 & 35 & 775 & 16 \\
\hline 29.1 & 52.99 & 67.13 & 1.27 & 14.58 & 0.0681 & 0.0068 & 1.1542 & 0.1016 & 0.1275 & 0.0033 & 779 & 48 & 774 & 19 \\
\hline 30.1 & 113.69 & 07.81 & 0.95 & 26.71 & 0.0635 & 0.0048 & 1.1571 & 0.0858 & 0.1287 & 0.0027 & 781 & 40 & 780 & 16 \\
\hline 31.1 & 107.45 & 108.03 & 1.01 & .36 & 0.0696 & 0.0045 & 1.2104 & 0.0751 & 0.1251 & 0.00 & 805 & & 760 & 16 \\
\hline 31.2 & 372.62 & 697.42 & 1.87 & 137.19 & 0.0660 & 0.0047 & 1.1348 & 0.0796 & 0.1273 & 0.0038 & 770 & 38 & 773 & 22 \\
\hline 33.1 & 109.59 & 93.17 & 0.85 & 25.07 & 0.0697 & 0.0045 & 1.2576 & 0.0772 & 0.1342 & 0.0036 & 827 & 35 & 812 & 20 \\
\hline 34.1 & 54.42 & 92.31 & 1.70 & & 0.0638 & 0.0056 & 1.1549 & 0.0983 & & & 780 & 46 & 786 & 17 \\
\hline 35.1 & 99.72 & 84.74 & & & 0.0665 & 0.0043 & 1.1971 & 0.0756 & & 0.0029 & 799 & 35 & 799 & 17 \\
\hline 36.1 & 146.48 & 135.30 & 0.92 & 33.05 & 0.0636 & 0.0042 & 1.1068 & 0.0678 & 0.1266 & 0.0026 & 757 & 33 & 768 & 15 \\
\hline 37.1 & 176.07 & 185.99 & 1.06 & 43.82 & 0.0670 & 0.0035 & 1.2750 & 0.0674 & 0.1378 & 0.0028 & 835 & 30 & 832 & 16 \\
\hline 38.1 & 88.98 & 75.69 & 0.85 & 8.80 & 0.0642 & 0.0044 & 1.1402 & 0.0769 & 0.1280 & 0.0026 & 773 & 37 & 776 & 15 \\
\hline 39.1 & 101.14 & 101.50 & 1.00 & 27.99 & 0.0681 & 0.0043 & 1.3604 & 0.0857 & 0.1445 & 0.0027 & 872 & 37 & 870 & 15 \\
\hline 41.1 & 56.66 & 54.94 & 0.97 & 12.79 & 0.0650 & 0.0054 & 1.0947 & 0.0888 & 0.1224 & 0.0028 & 751 & 43 & 744 & 16 \\
\hline 41.2 & 61.26 & 111.43 & 1.82 & 21.85 & 0.0659 & 0.0077 & 1.1505 & 0.1163 & 0.1282 & 0.0034 & 777 & 55 & 777 & 20 \\
\hline 43.1 & 85.73 & 132.48 & 1.55 & 26.80 & 0.0650 & 0.0052 & 1.1947 & 0.0863 & 0.1354 & 0.0029 & 798 & 40 & 819 & 16 \\
\hline 44.1 & 108.80 & 99.42 & 0.91 & 24.59 & 0.0655 & 0.0049 & 1.1854 & 0.0876 & 0.1311 & 0.0028 & 794 & 41 & 794 & 16 \\
\hline 45.1 & 60.11 & 83.77 & 1.39 & 17.15 & 0.0659 & 0.0053 & 1.2057 & 0.1030 & 0.1318 & 0.0040 & 803 & 47 & 798 & 23 \\
\hline 46.1 & 71.79 & 89.73 & 1.25 & 19.62 & 0.0619 & 0.0051 & 1.0959 & 0.0796 & 0.1311 & 0.0030 & 751 & 39 & 794 & 17 \\
\hline 48.1 & 61.17 & 49.91 & 0.82 & 14.41 & 0.0656 & 0.0053 & 1.3427 & 0.1216 & 0.1466 & 0.0046 & 864 & 53 & 882 & 26 \\
\hline
\end{tabular}

\subsection{Geochemical characteristics of the major elements}

\subsubsection{Characteristics and comparison of the chemical components}

\section{(1) LWZ Fm and QGZ Fm}

The data were divided into three groups: the LWZ Fm, QGZ Fm and Fajiaying Formation (FJY Fm). However, the FJY Fm had only one sample, which is not representative. The samples in the first two groups were carefully selected and included more than three samples, and the test results are of relatively high scientific value. The sandstones of LWZ Fm all show medium- and fine-grained clastic texture, moderate sorted or poor sorted and fair roundness - mainly of angular and subangular. The clastic constituents are composed of quartz (27-61 vol\%), feldspar (3-30 vol\%), debris (15-70 vol\%) and minor muscovite and accessory minerals. 
Table 4. LA-ICP-MS U-Pb zircon age data for the Proterozoic granitic gneiss and Mesozoic intrusive rocks in the Jiaonan Orogen.

\begin{tabular}{|c|c|c|c|c|c|c|c|c|c|c|c|c|c|c|}
\hline \multirow[b]{2}{*}{ Spot } & \multirow[b]{2}{*}{$\begin{array}{c}\mathrm{U} \\
(\mathrm{ppm})\end{array}$} & \multirow[b]{2}{*}{$\begin{array}{c}\text { Th } \\
(\mathrm{ppm})\end{array}$} & \multirow[b]{2}{*}{$\mathrm{Th} / \mathrm{U}$} & \multirow[b]{2}{*}{$\begin{array}{c}\mathrm{Pb}(\text { total }) \\
\quad(\mathrm{ppm})\end{array}$} & \multicolumn{5}{|c|}{ Isotopic ratios } & \multirow[b]{2}{*}{$1 \sigma$} & \multicolumn{4}{|c|}{ Age (Ma) } \\
\hline & & & & & $\begin{array}{c}{ }^{207} \mathrm{~Pb} / \\
{ }^{206} \mathrm{~Pb}\end{array}$ & $1 \sigma$ & $\begin{array}{c}{ }^{207} \mathrm{~Pb} / \\
{ }^{235} \mathrm{U}\end{array}$ & $1 \sigma$ & $\begin{array}{c}{ }^{206} \mathrm{~Pb} / \\
{ }^{238} \mathrm{U}\end{array}$ & & $\begin{array}{c}{ }^{207} \mathrm{~Pb} / \\
{ }^{235} \mathrm{U}\end{array}$ & $1 \sigma$ & $\begin{array}{c}{ }^{206} \mathrm{~Pb} / \\
{ }^{238} \mathrm{U}\end{array}$ & $1 \sigma$ \\
\hline \multicolumn{15}{|c|}{ Sample 10RZ-14 $\left(\mathrm{E} 119^{\circ} 5^{\prime} 55.5^{\prime \prime}, \mathrm{N} 35^{\circ} 27^{\prime} 57^{\prime \prime}\right),{ }^{206} \mathrm{~Pb} /{ }^{238} \mathrm{U}$ weighted mean age: $771 \pm 6.4 \mathrm{Ma}(\mathrm{MSWD}=0.21)$} \\
\hline 2.1 & 89.2 & 149 & 1.67 & 18.1 & 0.0865 & 0.0033 & 1.5096 & 0.0577 & 0.1264 & 0.0016 & 934 & 23 & 767 & 9 \\
\hline 3.1 & 83.9 & 79.1 & 0.94 & 14.6 & 0.0703 & 0.0030 & 1.2388 & 0.0522 & 0.1275 & 0.0015 & 818 & 24 & 774 & 9 \\
\hline 4.1 & 93.2 & 99.2 & 1.06 & 16.3 & 0.0667 & 0.0028 & 1.1614 & 0.0452 & 0.1268 & 0.0014 & 783 & 21 & 770 & 8 \\
\hline 5.1 & 99.3 & 118 & 1.19 & 17.7 & 0.0632 & 0.0026 & 1.0980 & 0.0456 & 0.1262 & 0.0015 & 752 & 22 & 766 & 9 \\
\hline 6.1 & 57.6 & 75.5 & 1.31 & 16.4 & 0.0672 & 0.0031 & 1.1827 & 0.0570 & 0.1276 & 0.0020 & 793 & 27 & 774 & 11 \\
\hline 7.1 & 44.3 & 56.6 & 1.28 & 16.5 & 0.0668 & 0.0041 & 1.1456 & 0.0675 & 0.1257 & 0.0019 & 775 & 32 & 764 & 11 \\
\hline 9.1 & 125 & 157 & 1.25 & 10.6 & 0.0632 & 0.0023 & 1.1078 & 0.0402 & 0.1273 & 0.0014 & 757 & 19 & 772 & 8 \\
\hline 15.1 & 71.4 & 84.6 & 1.18 & 15.3 & 0.0599 & 0.0035 & 1.0488 & 0.0577 & 0.1280 & 0.0017 & 728 & 29 & 777 & 10 \\
\hline \multicolumn{15}{|c|}{ Sample JDW-11 (E119 $\left.15^{\prime} 10.6^{\prime \prime}, \mathrm{N} 35^{\circ} 43^{\prime} 12^{\prime \prime}\right),{ }^{206} \mathrm{~Pb} /{ }^{238} \mathrm{U}$ weighted mean age: $751 \pm 6.3 \mathrm{Ma}(\mathrm{MSWD}=1.9)$} \\
\hline 1.1 & 97.8 & 93.03 & 1.05 & 16.2 & 0.0680 & 0.0034 & 1.1673 & 0.0553 & 0.1246 & 0.0018 & 785 & 26 & 757 & 10 \\
\hline 2.1 & 250.8 & 207.24 & 1.21 & 39.3 & 0.0680 & 0.0013 & 1.1553 & 0.0234 & 0.1233 & 0.0016 & 780 & 11 & 749 & 9 \\
\hline 3.1 & 185.1 & 193.31 & 0.96 & 30.6 & 0.0681 & 0.0014 & 1.1742 & 0.0260 & 0.1251 & 0.0016 & 789 & 12 & 760 & 9 \\
\hline 4.1 & 279.5 & 923.36 & 0.30 & 48.5 & 0.0674 & 0.0013 & 1.1564 & 0.0240 & 0.1244 & 0.0016 & 780 & 11 & 756 & 9 \\
\hline 5.1 & 196.0 & 185.88 & 1.05 & 30.7 & 0.0660 & 0.0015 & 1.1049 & 0.0260 & 0.1214 & 0.0016 & 756 & 13 & 739 & 9 \\
\hline 6.1 & 72.6 & 78.11 & 0.93 & 12.1 & 0.0644 & 0.0023 & 1.1180 & 0.0405 & 0.1259 & 0.0017 & 762 & 19 & 764 & 10 \\
\hline 7.1 & 156.8 & 130.79 & 1.20 & 24.5 & 0.0667 & 0.0016 & 1.1525 & 0.0286 & 0.1253 & 0.0016 & 778 & 14 & 761 & 9 \\
\hline 8.1 & 393.3 & 582.25 & 0.68 & 69.9 & 0.0658 & 0.0013 & 1.1188 & 0.0230 & 0.1234 & 0.0016 & 762 & 11 & 750 & 9 \\
\hline 9.1 & 206.0 & 209.14 & 0.99 & 33.4 & 0.0674 & 0.0014 & 1.1551 & 0.0256 & 0.1242 & 0.0016 & 780 & 12 & 755 & 9 \\
\hline 10.1 & 165.4 & 179.12 & 0.92 & 27.7 & 0.0642 & 0.0034 & 1.0946 & 0.0564 & 0.1237 & 0.0018 & 751 & 27 & 752 & 10 \\
\hline 11.1 & 121.0 & 132.68 & 0.91 & 19.6 & 0.0663 & 0.0020 & 1.1013 & 0.0332 & 0.1205 & 0.0016 & 754 & 16 & 734 & 9 \\
\hline 12.1 & 147.2 & 193.37 & 0.76 & 25.3 & 0.0661 & 0.0018 & 1.1244 & 0.0310 & 0.1234 & 0.0016 & 765 & 15 & 750 & 9 \\
\hline 13.1 & 156.5 & 191.92 & 0.82 & 26.2 & 0.0650 & 0.0038 & 1.0715 & 0.0602 & 0.1196 & 0.0018 & 739 & 30 & 728 & 10 \\
\hline 14.1 & 171.1 & 172.28 & 0.99 & 27.3 & 0.0645 & 0.0016 & 1.0937 & 0.0285 & 0.1231 & 0.0016 & 750 & 14 & 748 & 9 \\
\hline 15.1 & 205.3 & 141.96 & 1.45 & 33.3 & 0.0667 & 0.0033 & 1.1275 & 0.0539 & 0.1226 & 0.0017 & 767 & 26 & 745 & 10 \\
\hline 16.1 & 103.8 & 93.41 & 1.11 & 16.8 & 0.0641 & 0.0035 & 1.1135 & 0.0581 & 0.1259 & 0.0018 & 760 & 28 & 764 & 10 \\
\hline 17.1 & 140.4 & 153.94 & 0.91 & 23.9 & 0.0681 & 0.0017 & 1.2178 & 0.0309 & 0.1296 & 0.0017 & 809 & 14 & 786 & 10 \\
\hline 18.1 & 55.9 & 60.96 & 0.92 & 9.1 & 0.0618 & 0.0032 & 1.0579 & 0.0546 & 0.1242 & 0.0017 & 733 & 27 & 755 & 10 \\
\hline 19.1 & 197.8 & 182.94 & 1.08 & 30.3 & 0.0664 & 0.0016 & 1.0990 & 0.0266 & 0.1201 & 0.0016 & 753 & 13 & 731 & 9 \\
\hline
\end{tabular}

Sample 10RZ-20 $\left(\mathrm{E} 119^{\circ} 5^{\prime} 27^{\prime \prime}, \mathrm{N} 35^{\circ} 23^{\prime} 2^{\prime \prime}\right),{ }^{206} \mathrm{~Pb} /{ }^{238} \mathrm{U}$ weighted mean age: $126 \pm 3.0 \mathrm{Ma}(\mathrm{MSWD}=2.9)$

\begin{tabular}{|c|c|c|c|c|c|c|c|c|c|c|c|c|c|}
\hline 1.1 & 24.0 & 31.6 & 1.32 & 0.6 & 0.0484 & 0.0225 & 0.1218 & 0.0566 & 0.0183 & 0.0006 & 117 & 51 & 117 \\
\hline 2.1 & 44.3 & 66.6 & 1.50 & 1.2 & 0.0483 & 0.0235 & 0.1274 & 0.0617 & 0.0191 & 0.0006 & 122 & 56 & 122 \\
\hline 3.1 & 51.2 & 93.5 & 1.83 & 1.5 & 0.0485 & 0.0157 & 0.1255 & 0.0405 & 0.0188 & 0.0005 & 120 & 36 & 120 \\
\hline 4.1 & 26.1 & 34.3 & 1.31 & 0.7 & 0.0487 & 0.0339 & 0.1307 & 0.0909 & 0.0195 & 0.0008 & 125 & 82 & 124 \\
\hline 5.1 & 31.7 & 44.3 & 1.40 & 1.2 & 0.1069 & 0.0415 & 0.2711 & 0.1042 & 0.0184 & 0.0010 & 244 & 83 & 118 \\
\hline 6.1 & 56.5 & 72.8 & 1.29 & 1.5 & 0.0484 & 0.0120 & 0.1323 & 0.0328 & 0.0198 & 0.0004 & 126 & 29 & 126 \\
\hline 7.1 & 26.7 & 37.4 & 1.40 & 1.7 & 0.0571 & 0.0272 & 0.1367 & 0.0648 & 0.0174 & 0.0006 & 130 & 58 & 111 \\
\hline 8.1 & 49.3 & 67.9 & 1.38 & 1.3 & 0.0486 & 0.0255 & 0.1266 & 0.0663 & 0.0189 & 0.0007 & 121 & 60 & 121 \\
\hline 9.1 & 45.2 & 78.5 & 1.74 & 1.4 & 0.0641 & 0.0227 & 0.1809 & 0.0638 & 0.0205 & 0.0006 & 169 & 55 & 131 \\
\hline 10.1 & 41.2 & 83.3 & 2.02 & 1.2 & 0.0484 & 0.0217 & 0.1277 & 0.0573 & 0.0192 & 0.0006 & 122 & 52 & 122 \\
\hline 11.1 & 68.4 & 134.9 & 1.97 & 2.1 & 0.0486 & 0.0079 & 0.1348 & 0.0218 & 0.0201 & 0.0004 & 128 & 19 & 128 \\
\hline 13.1 & 27.7 & 44.5 & 1.61 & 1.0 & 0.0488 & 0.0353 & 0.1482 & 0.1072 & 0.0220 & 0.0008 & 140 & 95 & 141 \\
\hline 14.1 & 59.8 & 95.8 & 1.60 & 1.7 & 0.0488 & 0.0079 & 0.1388 & 0.0223 & 0.0206 & 0.0004 & 132 & 20 & 132 \\
\hline 15.1 & 71.7 & 142.3 & 1.99 & 1.5 & 0.0484 & 0.0078 & 0.1303 & 0.0210 & 0.0195 & 0.0004 & 124 & 19 & 125 \\
\hline 16.1 & 32.8 & 43.3 & 1.32 & 0.9 & 0.0487 & 0.0200 & 0.1356 & 0.0555 & 0.0202 & 0.0006 & 129 & 50 & 129 \\
\hline 17.1 & 39.2 & 62.9 & 1.61 & 1.1 & 0.0485 & 0.0131 & 0.1361 & 0.0368 & 0.0204 & 0.0005 & 130 & 33 & 130 \\
\hline 18.1 & 35.8 & 51.3 & 1.43 & 1.0 & 0.0490 & 0.0161 & 0.1360 & 0.0446 & 0.0201 & 0.0006 & 129 & 40 & 128 \\
\hline
\end{tabular}



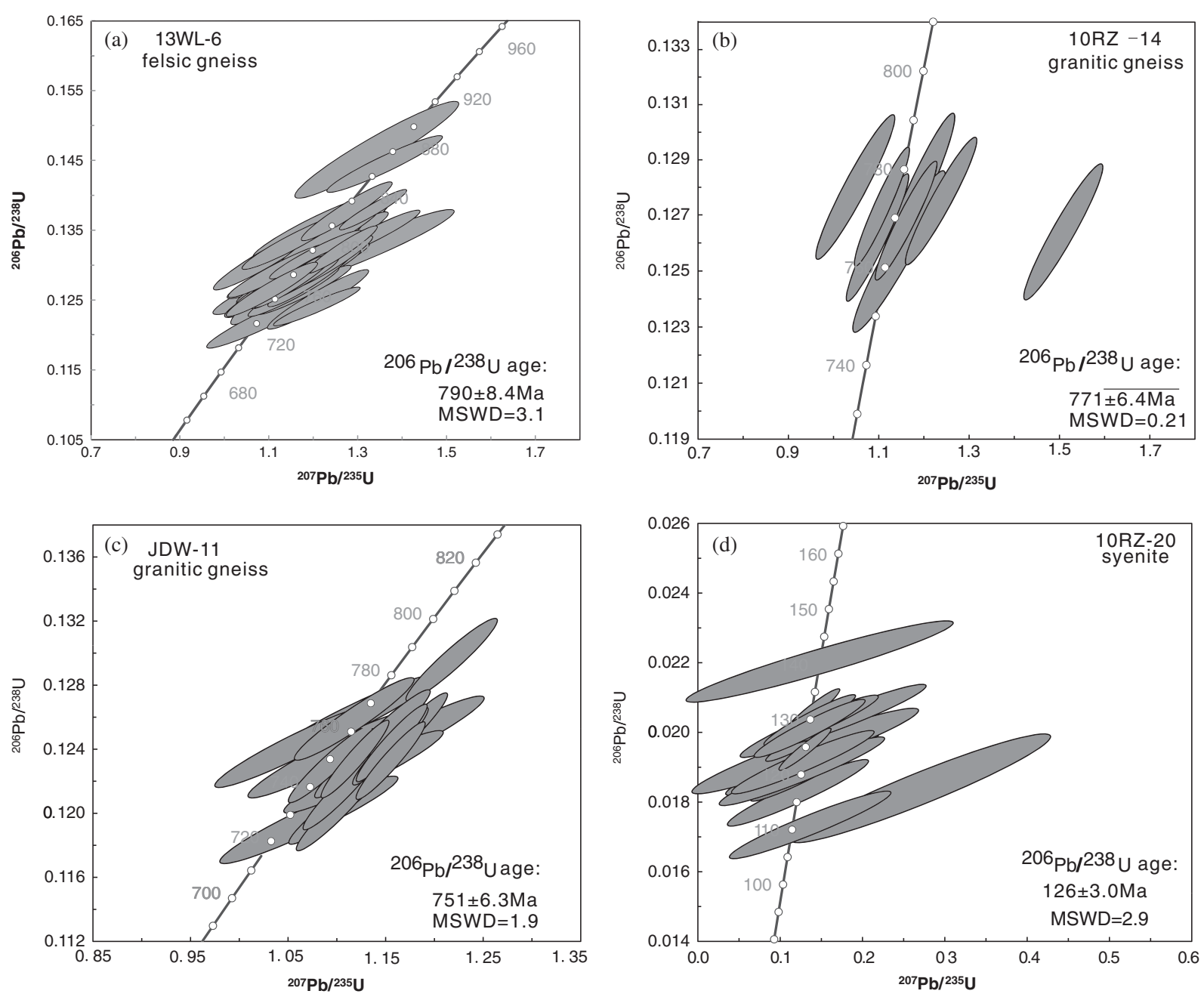

Figure 5. Zircon U-Pb concordia diagram of (a) the granitic gneiss at the base of the Laiyang Group's conglomerates, (b and $\mathbf{c})$ the Neoproterozoic granites in the Jiaonan Orogen, and (d) the Mesozoic syenite in the Jiaonan Orogen.

The sandstones of QGZ Fm are similar to those of LWZ Fm, and all of medium and fine grained clastic texture, poor sorted and of poor roundness - with obvious angles. The sandstones of QGZ Fm consisted of quartz (30-57 vol\%), feldspar (15-57 vol\%), debris (8-55 vol\%) and minor muscovite and heavy minerals.

The LWZ Fm has a higher $\mathrm{SiO}_{2}$ content (average 72.87 wt.\%) than the QGZ Fm (average 70.26 wt.\%), but the other major element contents, apart from $\mathrm{CaO}$, are lower in the former than in the latter (table 1). The $\mathrm{Al}_{2} \mathrm{O}_{3}$ content in the LWZ Fm is between 11.83 and 12.48 wt.\%, averaging 12.11 wt.\%; the $\mathrm{K}_{2} \mathrm{O}$ content is between 1.94 and 2.63 wt.\%, averaging 2.16 wt.\%; the $\mathrm{Na}_{2} \mathrm{O}$ content is between 2.88 and 4.55 wt.\%, averaging 3.56 wt.\%; the $\mathrm{Fe}_{2} \mathrm{O}_{3}^{\mathrm{T}}+\mathrm{MgO}$ content averages 3.74 wt.\%; and the $\mathrm{TiO}_{2}$ content is between 0.37 and 0.56 wt. $\%$, averaging 0.46 wt.\%. The $\mathrm{Al}_{2} \mathrm{O}_{3}$ content in the QGZ Fm is between 12.02 and 16.17 wt.\%, averaging 13.93 wt. $\%$; the $\mathrm{K}_{2} \mathrm{O}$ content is between 1.81 and 3.48 wt.\%, averaging
2.47 wt.\%; the $\mathrm{Na}_{2} \mathrm{O}$ content is between 2.52 and 6.27 wt.\%, averaging 4.34 wt.\%; and the $\mathrm{Fe}_{2} \mathrm{O}_{3}^{\mathrm{T}}+\mathrm{MgO}$ content averages 5.84 wt. $\%$.

Comparing the major element data of the LWZ Fm and QGZ Fm with the previously studied average (Bhatia and Crook 1986) chemical compositions of ocean island sandstones, continental island arc sandstones, active continental margins and passive continental margins (table 2), the characteristics of the LWZ Fm in the lower Laiyang Group are most similar to those of active continental margin sandstones, whereas those of the QGZ Fm in the upper Laiyang Group are most similar to those of continental island arcs.

(2) Comparison of the Laiyang Group with the Proterozoic and Mesozoic intrusive rocks

We also compared the major element compositions of the Laiyang Group's sandstones (including LWZ, QGZ and FJY Formations) to those of the Neoproterozoic granitic gneisses in the Jiaonan Orogen (table 1, figure 6). The $\mathrm{TiO}_{2}, \mathrm{Fe}_{2} \mathrm{O}_{3}^{\mathrm{T}}, \mathrm{MnO}$ and 

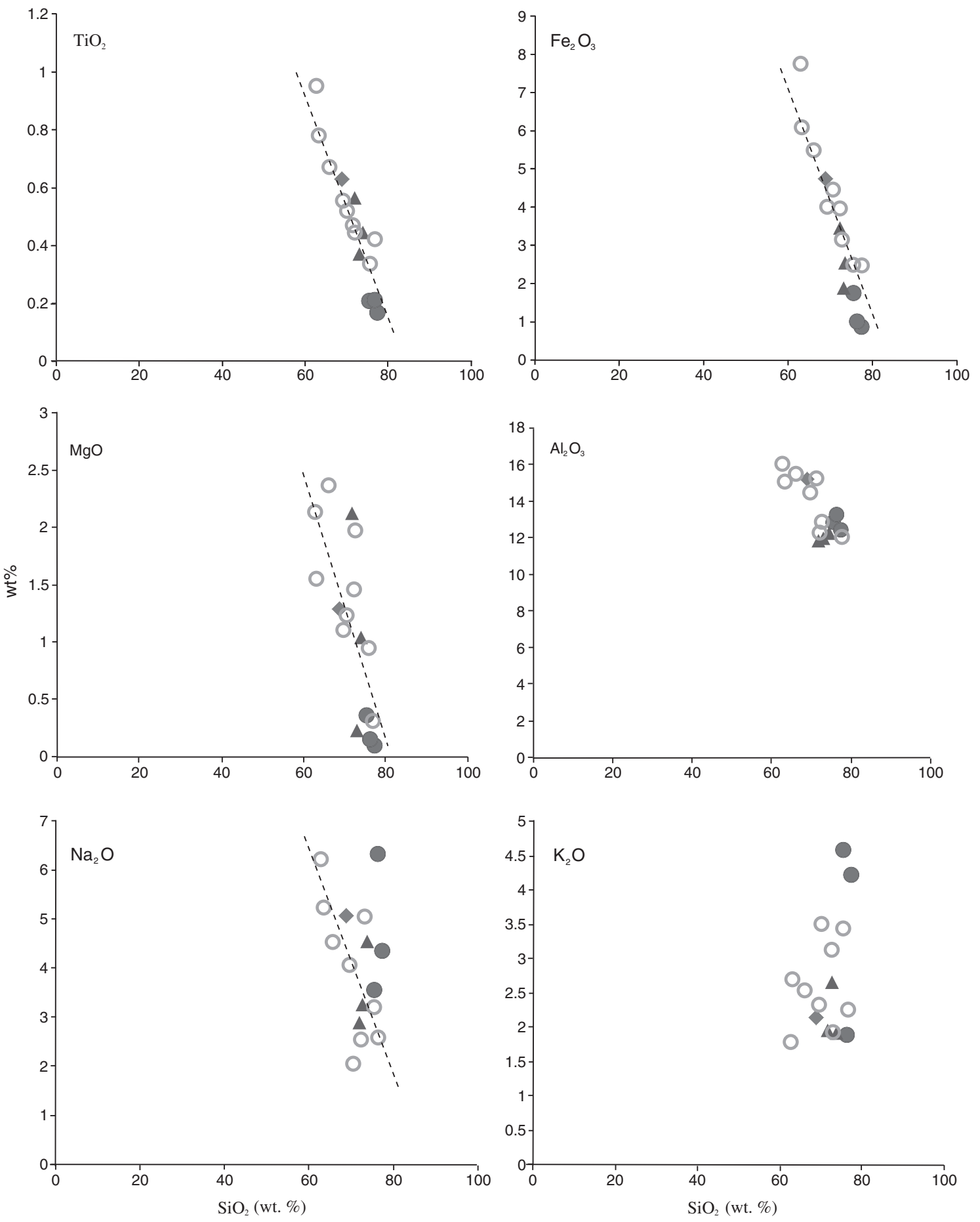

LEGEND

$\Delta$ LWZ Formation $\bigcirc$ QGZ Formation

$\checkmark$ FJY Formation

Proterozoic granite

Figure 6. Hacker variable diagram of the Laiyang Group's sandstones from the Zhucheng Basin.

$\mathrm{MgO}$ contents of the Laiyang Group's sandstones are markedly higher than in the Neoproterozoic granitic gneisses, whereas the $\mathrm{SiO}_{2}$ and $\mathrm{K}_{2} \mathrm{O}$ contents are lower in the former than in the latter. The other major element concentrations are similar (table 1, figure 6). The $\mathrm{SiO}_{2}$ concentrations of the Laiyang Group's sandstones are higher than those of the Mesozoic syenite in the Jiaonan
Orogen, but the other major element concentrations are all lower (table 1 ).

(3) Factors influencing the chemical compositions of the sandstones

Correlations exist between the quartz grains, feldspar debris and unstable rock debris present in sandstones and between the various elements in 
the sandstones (Bhatia 1983). Hence, this paper studied the correlations between the major element geochemical data from the LWZ Fm, QGZ Fm and Neoproterozoic granitic gneisses (figure 6).

The $\mathrm{SiO}_{2}$ content exhibits an obvious negative linear correlation with $\mathrm{TiO}_{2}, \mathrm{Fe}_{2} \mathrm{O}_{3}, \mathrm{MgO}$, and $\mathrm{Na}_{2} \mathrm{O}$ in the sandstones of each formation in the study area (figure 6). The plot of the Neoproterozoic granitic gneiss samples and the plot of the sandstone samples are on the same oblique line, indicating that the quartz grains in the sandstones and the silicate minerals in the granitic gneiss influence the chemical properties of the whole rock to a great extent. This plot also highlights the similar compositions of the source regions of the LWZ Fm and QGZ Fm. However, the correlations of $\mathrm{SiO}_{2}$ with $\mathrm{Al}_{2} \mathrm{O}_{3}$ and $\mathrm{K}_{2} \mathrm{O}$ are not clear (figure 5), which may be attributed to residual $\mathrm{Al}_{2} \mathrm{O}_{3}$ minerals and the loss of $\mathrm{K}$ ions due to weathering in the source region.

\subsubsection{Types of sandstones}

Sandstones are generally geochemically classified by the major element oxide concentrations. For example, the $\mathrm{SiO}_{2} / \mathrm{Al}_{2} \mathrm{O}_{3}$ ratio can distinguish quartz arenite with abundant silicon from mudstones with abundant aluminium (Herron 1988), and this ratio can also reflect constituent enrichments in quartz, clay minerals and feldspar (Cox and Lowe 1995). The $\mathrm{Fe}_{2} \mathrm{O}_{3} / \mathrm{K}_{2} \mathrm{O}$ ratio can distinguish lithic sandstones from feldspar sandstones (arkoses and subarkose), which can indicate the stability of mafic minerals in sandstones during the course of weathering (Heron 1988). The $\mathrm{K}_{2} \mathrm{O} / \mathrm{Na}_{2} \mathrm{O}$ ratio can reflect chemical maturity (Pettijohn et al. 1972). Previous researchers have established the geochemical classification of sandstones according to these ratios (Herron 1988) (figure 6).

For the LWZ Fm and QGZ Fm, the average $\mathrm{SiO}_{2} / \mathrm{Al}_{2} \mathrm{O}_{3}$ ratios are 6.02 and 5.04, respectively;
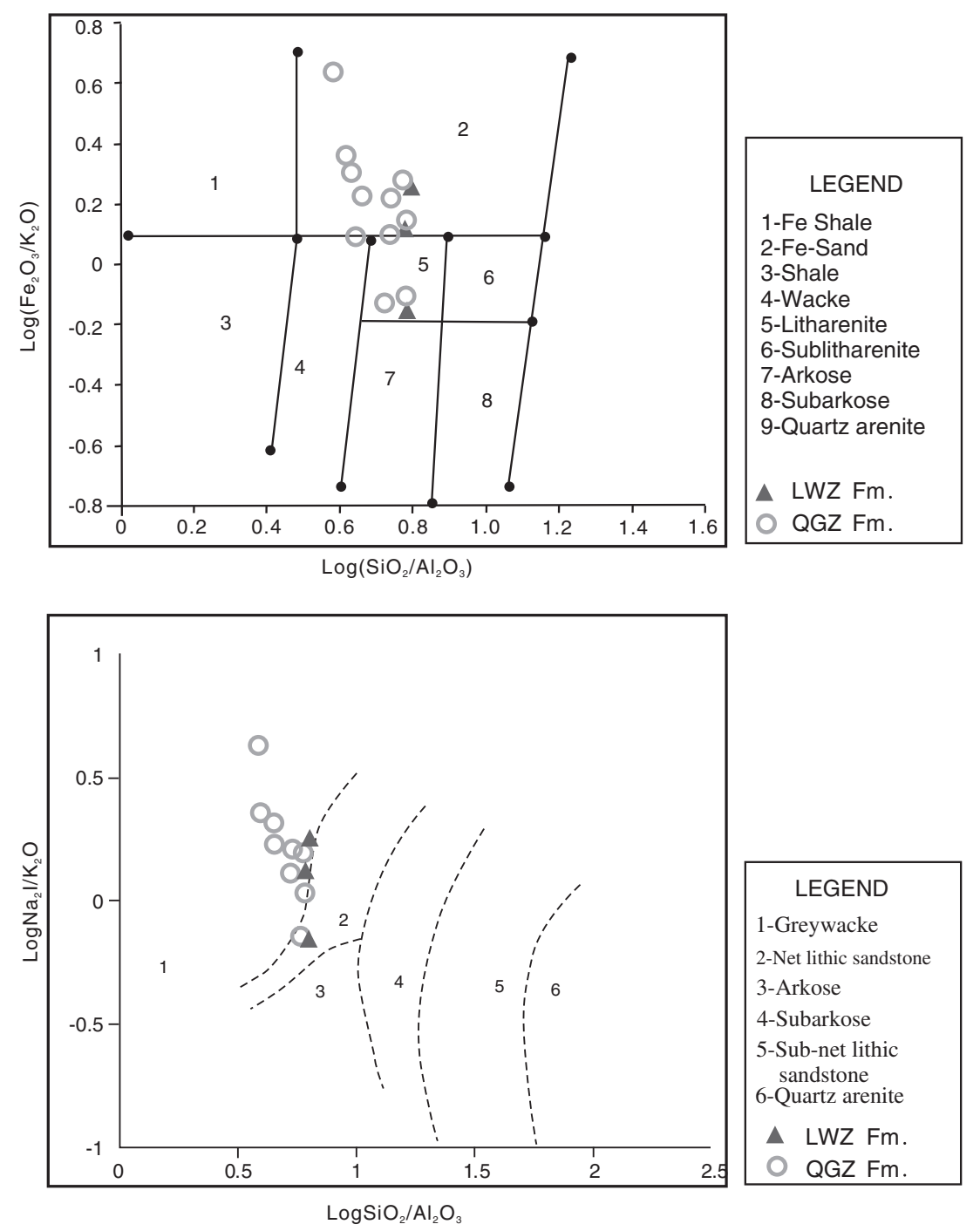

Figure 7. Geochemical classification diagram (Herron 1988) of the major elements of the Laiyang Group's sandstones from the Zhucheng Basin. 
the $\mathrm{Fe}_{2} \mathrm{O}_{3} / \mathrm{K}_{2} \mathrm{O}$ ratios are 1.21 and 1.76 , respectively; and the $\mathrm{K}_{2} \mathrm{O} / \mathrm{Na}_{2} \mathrm{O}$ ratios are 0.63 and 0.65 , respectively (table 1$)$. In the $\log \left(\mathrm{SiO}_{2} / \mathrm{Al}_{2} \mathrm{O}_{3}\right)_{-}$ $\log \left(\mathrm{Fe}_{2} \mathrm{O}_{3} / \mathrm{K}_{2} \mathrm{O}\right)$ classification diagram, most of the sample points fall in the $\mathrm{Fe}$ sandstone area (figure 7a). In the $\log \left(\mathrm{SiO}_{2} / \mathrm{Al}_{2} \mathrm{O}_{3}\right)-\log \left(\mathrm{Na}_{2} \mathrm{O} / \mathrm{K}_{2} \mathrm{O}\right)$ classification diagram, most of the sample points fall in the greywacke area, with a small number of sample points falling in the lithic sandstone area (figure 7b). These results suggest that the sandstones in the Zhucheng Basin did not experience intense weathering and were deposited near the source area or experienced rapid diagenesis. Additionally, abundant mafic components may be present in the source area.

\subsubsection{Sandstone chemical alteration and source area paleo-weathering}

During the course of rock weathering, deposition and diagenesis, the oxide concentrations of unstable elements (such as $\mathrm{CaO}, \mathrm{Na}_{2} \mathrm{O}, \mathrm{K}_{2} \mathrm{O}$, and $\mathrm{MgO}$ ) and relatively stable elements (such as $\mathrm{Al}_{2} \mathrm{O}_{3}$, $\mathrm{ZrO}_{2}$, and $\mathrm{TiO}_{2}$ ) can change (Cox et al. 1995). Several indices of sandstone chemical alteration and paleo-weathering based on the major element oxide contents have been proposed by previous researchers. Frequently used indicators include the Chemical Index of Alteration (CIA) (McLennan et al. 1993), the Chemical Index of Weathering (CIW) (Harnois 1988), the Plagioclase Index of Alteration (PIA) (Fedo et al. 1995) and the Index of Chemical Constituent Variation (ICV) (Cox et al. 1995) (table 5).

Based on these indices, similarities and differences exist between the LWZ Fm and QGZ Fm. Their ICV average values are 0.99 and 1.01, respectively, which are close to 1.0 and do not differ significantly. Generally, first-cycle sediments are characterised by the presence of less resistant minerals and rock fragments. If this material is reworked through a second cycle, the less resistant minerals will be eliminated, or altered to more stable products. When the ICV values are greater than 1.0, the sandstones are likely first-cycle sediments, whereas ICV values less than 1.0 indicate that the sandstones are likely recycled sediments or first-cycle sediments that have experienced intense weathering (Cox et al. 1995). The ICV values of the LWZ Fm are between 0.95 and 1.02, suggesting that the source area did not experience intense weathering. The ICV values of the QGZ Fm are between 0.86 and 1.30 , indicating that great variations in the degree of weathering are present in the source rocks of the QGZ Fm; certain source rocks were relatively intensely weathered, whereas others were not.
All the other indices for the LWZ Fm are lower than those for the QGZ Fm. For instance, the LWZ Fm's CIA (average: 47 ) and CIW (average: 51 ) values are lower than those of the QGZ Fm (50 and 5, respectively). Previous researchers have considered CIA values between 45 and 55 to indicate no weathering, whereas a value of 100 indicates intense weathering and the complete loss of alkaline-earth metals (such as Ca, Mg and $\mathrm{Sr}$ ) (McLennan 1993; Jason and Michale 2003). The average value of the upper crust is 47 (McLennan 1993). Therefore, the weathering in the source areas of the LWZ Fm and QGZ Fm was very weak, but the weathering of the latter was slightly more intense than that of the former, which is also reflected by the PIA. The PIA average values of the QGZ Fm and LWZ Fm are 64 and 60, respectively. Generally, the PIA values are about 50 for fresh rocks and values close to 100 for clay minerals such as kaolinite, illite, and gibbsite (Fedo et al. 1995). These data suggest that the source area is nearby the Zhucheng Basin, and the rock in the source area has not undergone intense weathering, which indicates the intense uplift occurred in the source area of the Zhucheng Basin. Furthermore, the source area of the LWZ Fm likely rose more rapidly during the deposition of the LWZ Fm than that of the QGZ $\mathrm{Fm}$ because the CIA value is similar to the average value of the upper crust and suggests the lack of apparent weathering in the source area. The above

Table 5. Chemical alteration and weathering features of the LWZ Fm and QGZ Fm from the Zhucheng Basin.

\begin{tabular}{lllll}
\hline Sample no. & CIA & CIW & PIA & ICV \\
\hline LWZ Fm & & & & \\
JDW-71 & 45 & 48 & 58 & 1.02 \\
JDW-74 & 49 & 54 & 63 & 0.99 \\
JDW-82 & 46 & 52 & 59 & 0.95 \\
Average & $\mathbf{4 7}$ & $\mathbf{5 1}$ & $\mathbf{6 0}$ & $\mathbf{0 . 9 9}$ \\
QGZ Fm & & & & \\
JDW-28 & 51 & 55 & 64 & 1.15 \\
JDW-29 & 52 & 57 & 65 & 1.01 \\
JDW-77 & 43 & 47 & 55 & 1.30 \\
JDW-78 & 58 & 69 & 73 & 0.76 \\
JDW-79 & 48 & 52 & 61 & 0.99 \\
JDW-80 & 48 & 52 & 61 & 0.99 \\
JDW-81 & 50 & 58 & 64 & 1.03 \\
JDW-83 & 51 & 57 & 65 & 0.86 \\
JDW-85 & 49 & 54 & 63 & 0.97 \\
Average & $\mathbf{5 0}$ & $\mathbf{5 6}$ & $\mathbf{6 4}$ & $\mathbf{1 . 0 1}$ \\
\hline
\end{tabular}

Note: CIA $=\left[\mathrm{Al}_{2} \mathrm{O}_{3} /\left(\mathrm{CaO}^{*}+\mathrm{Na}_{2} \mathrm{O}+\mathrm{K}_{2} \mathrm{O}+\mathrm{Al}_{2} \mathrm{O}_{3}\right)\right] \times 100$ (McLennan 1993), CIW $=\left[\mathrm{Al}_{2} \mathrm{O}_{3} /\left(\mathrm{CaO}^{*}+\mathrm{Na}_{2} \mathrm{O}+\mathrm{Al}_{2} \mathrm{O}_{3}\right)\right] \times$ 100 (Harnois 1988), PIA $=\left[\left(\mathrm{Al}_{2} \mathrm{O}_{3}-\mathrm{K}_{2} \mathrm{O}\right) /\left(\mathrm{CaO}^{*}+\mathrm{Na}_{2} \mathrm{O}+\right.\right.$ $\left.\left.\mathrm{Al}_{2} \mathrm{O}_{3}-\mathrm{K}_{2} \mathrm{O}\right)\right] \times 100$ (Fedo et al. 1995), $\mathrm{ICV}=\left(\mathrm{Fe}_{2} \mathrm{O}_{3}+\mathrm{K}_{2} \mathrm{O}+\right.$ $\mathrm{Na} 2 \mathrm{O}+\mathrm{CaO}^{*}+\mathrm{MgO}+\mathrm{MnO}+\mathrm{TiO}_{2}$ )/ $\mathrm{Al}_{2} \mathrm{O}_{3}$ (Cox et al. 1995). 
conclusion is consistent with the high exhumed rate of $2.0 \mathrm{~km} \mathrm{Ma}{ }^{-1}$ from before c. 128 to $123 \mathrm{Ma}$ in Jiaonan Orogen (Ni et al. 2016).

The intensity and trend of the source area's weathering can also be shown on $\mathrm{A}-\mathrm{CN}-\mathrm{K}$ and A-CNK-FM diagrams (McLennan et al. 1993) (figure 8). The difference in these two diagrams lies in the consideration of the mafic components. Feldspar and alkaline alumino-silicate minerals are generally considered to transform into clay minerals during rock weathering, unstable oxides (such as $\mathrm{CaO}, \mathrm{Na}_{2} \mathrm{O}$ and $\mathrm{K}_{2} \mathrm{O}$ ) are easily lost, and stable oxides (such as $\mathrm{Al}_{2} \mathrm{O}_{3}$ ) increase proportionately (McLennan et al. 1993). As a result, if the rocks do not experience potassic alteration, the rock weathering trend will be parallel to the $\mathrm{A}-\mathrm{CN}$ edge in the $\mathrm{A}-\mathrm{CN}-\mathrm{K}$ diagram, expanding in the directions of kaolinite, illite, and white mica (figure 8).

All the sample points are plotted in the $\mathrm{A}-\mathrm{CN}-\mathrm{K}$ and $\mathrm{A}-\mathrm{CNK}-\mathrm{FM}$ diagrams. In the $\mathrm{A}-\mathrm{CN}-\mathrm{K}$ diagram, all the sample points are close to plagioclase, far from potassium feldspar. Additionally, majority of the sample points are under or in the vicinity of the plagioclase-potassium feldspar boundary, indicating that the plagioclase contents are higher than the potassium contents in the samples; thus, the weathering of feldspar in the rocks was very weak (CIA values mostly lower than $50 \%$ ). The rock weathering trends are generally oriented in the direction of illite (figure 8a) and are parallel to the $\mathrm{A}-\mathrm{CN}$ edge, suggesting that the rocks did not experience potassium metasomatism. The plotted points are close to granodiorite and andesite, consistent with the ferruginous sandstones in the geochemistry classification of the samples discussed above. The sample points are more disperse in the $\mathrm{A}-\mathrm{CNK}-\mathrm{FM}$ diagram (figure $8 \mathrm{~b}$ ) than in the $\mathrm{A}-\mathrm{CN}-\mathrm{K}$ diagram, because the samples contain variable quantities of mafic components. However, the sample points are still close to granodiorite and in the vicinity of the potassium feldspar-FM line, demonstrating that the rocks did not undergo obvious weathering, which is consistent with the results from the $\mathrm{A}-\mathrm{CN}-\mathrm{K}$ diagram.

\subsection{Trace element geochemistry \\ 4.3.1 LWZ Fm and QGZ Fm}

The trace element and REE contents of the Laiyang Group's sandstones are listed in table 2. The trace element contents of the Laiyang Group's sandstones in the Zhucheng Basin are lower than those of the upper continental crust (Rudnick and Gao 2003), with the exception of Rb, Zr and Ba, which are higher. In particular, $\mathrm{Co}, \mathrm{Ni}, \mathrm{Sr}$ and $\mathrm{Ta}$ are obviously lower than in the upper continental crust and trend towards acidity. The trace element contents in the LWZ Fm in the Lower Laiyang Group are obviously higher than those in the QGZ Fm in the Upper Laiyang Group, with the exception of $\mathrm{Cr}, \mathrm{Ni}$, Cs and $\mathrm{Ba}$.

In the primitive mantle-normalised spider diagram of the trace elements (figure 9), the Laiyang Group in the Zhucheng Basin exhibits overall enrichment in the large ion lithophile elements $\mathrm{Rb}, \mathrm{Ba}$ and $\mathrm{K}$ and REEs La and Ce; depletion in the inactive elements $\mathrm{P}, \mathrm{Ti}, \mathrm{Y}, \mathrm{Yb}$ and $\mathrm{Lu}$; intermediate concentrations of Zr and Hf; and variable Sr concentrations. The individual spider diagrams for the LWZ Fm and QGZ Fm (figure 9a) are generally similar, but the LWZ Fm curve is primarily below the QGZ Fm, with the exception of $\mathrm{Rb}, \mathrm{Ba}$, Th and U. This difference is more obvious in REEnormalised distribution pattern diagrams. On both the chondrite-normalised (figure 10a) and North

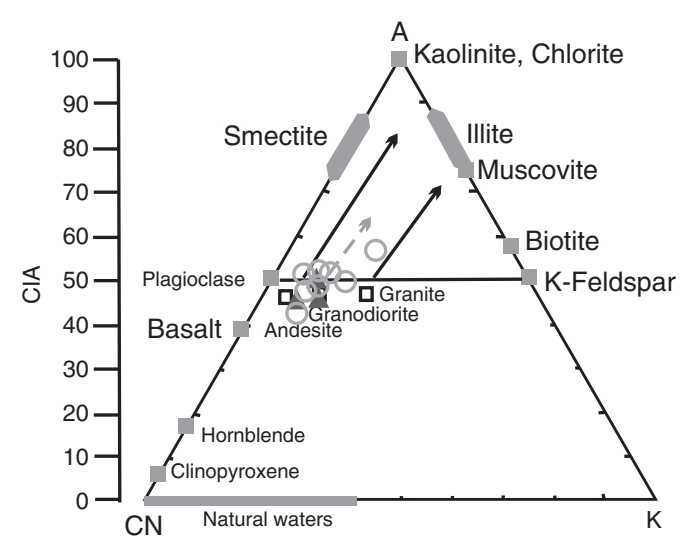

(a)

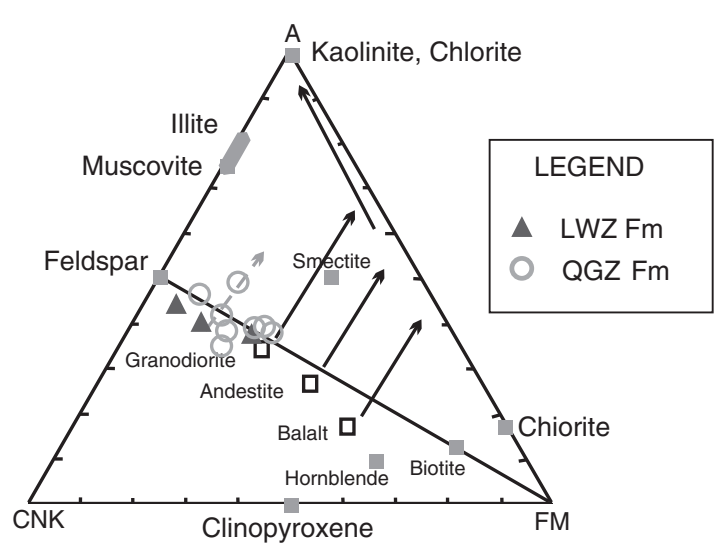

(b)

Figure 8. (a) A-CN-K and (b) A-CNK-FM diagrams of the major elements of the Laiyang Group's sandstones from the Zhucheng Basin (McLennan et al. 1993). Note: $\mathrm{A}=\mathrm{Al}_{2} \mathrm{O}_{3}, \mathrm{CN}=\mathrm{CaO}+\mathrm{Na}_{2} \mathrm{O}, \mathrm{K}=\mathrm{K}_{2} \mathrm{O}, \mathrm{FM}=\mathrm{Fe}_{2} \mathrm{O}_{3}+\mathrm{MgO}, \mathrm{CIA}=$ Chemical Index of Alteration. All the samples are calculated in mole numbers. 

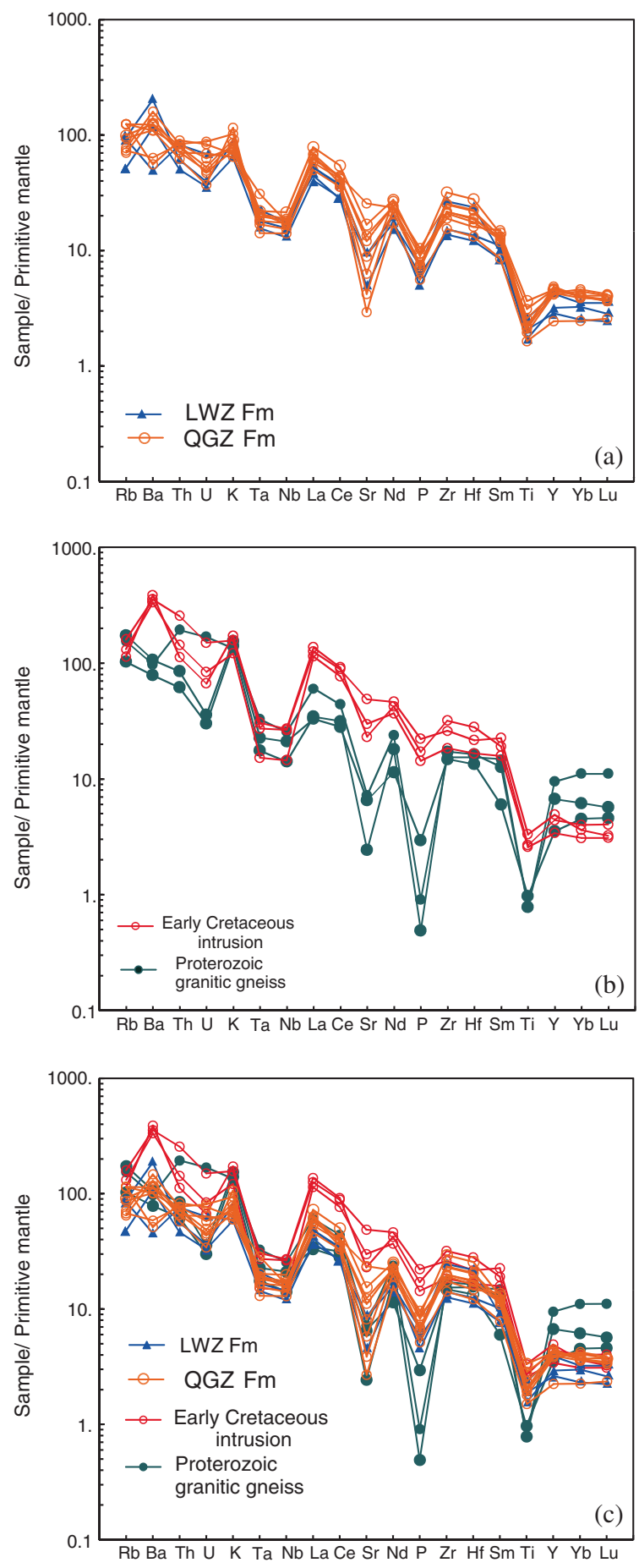

Figure 9. Primitive mantle-normalized spider diagram of the trace elements (normalized data derived from Sun and McDonough (1989)). (a) Laiyang Group's sandstones from the Zhucheng Basin and the Laiyang Depression; (b) intrusive rocks from the Jiaonan Orogen; and (c) comparison of the LWZ Fm with the Proterozoic intrusive rocks and several Mesozoic stocks.

American Shale Composite normalised diagrams (figure 10b), the sample curves of the QGZ Fm are primarily above those of the LWZ Fm, except for one unseparated broken line. This difference may indicate that the sediments experienced chemical element separation and migration during the deposition of these formations and that the elevation difference between the basin and provenance decreased during the sedimentation of the QGZ Fm. This process allowed sufficient time for the REEs in the sediments to become separated, and they were subsequently preserved in the basin.

Light REEs are enriched on the REE chondritenormalised diagram for the Laiyang Group's sandstones in the Zhucheng Basin (figure 10a). By contrast, the heavy REEs are relatively depleted, and weak negative Eu anomalies are present. The REE content of the Laiyang Group's sandstones is almost identical to that of the North American Shale Composite (figure 10b), with slight depletion in the heavy REEs and slightly higher Eu contents, indicating that the sandstones in the LWZ Fm and QGZ Fm in the Laiyang Group experienced a relatively complete sedimentary cycle. Critically, heavy earth elements are not enriched, demonstrating that the geochemical properties of the samples were not greatly affected by water-rock reactions that might diminish their ability to discriminate provenances and tectonic settings.

\subsubsection{Laiyang Group, Neoproterozoic granitic gneiss and Mesozoic intrusive rocks}

The curved shape of the trace element plot for the Laiyang Group from the Zhucheng Basin is similar to that of the Neoproterozoic granitic gneiss in the Jiaonan Orogen (figure 9a, b). For instance, the Neoproterozoic granitic gneisses are enriched in the large ion lithophile elements $\mathrm{Rb}, \mathrm{Ba}, \mathrm{U}$ and $\mathrm{K}$, the REEs La and Ce, and the elements $\mathrm{Y}, \mathrm{Yb}$ and $\mathrm{Lu}$, and they are depleted in typical inactive elements, such as Sr, P and Ti.

On the spider diagrams, the trace elements of the Laiyang Group are also similar to those of the syenite that intruded in the early Cretaceous, but the values of the former are slightly lower than those of the latter (figure 9c). Each value for the LWZ Fm is between that of the Neoproterozoic granitic gneiss and that of the Lower Cretaceous diorite.

In the REE chondrite-normalised diagrams, the light REE contents of the LWZ Fm are similar to those of the Neoproterozoic granitic gneisses, and both exhibit negative Eu anomalies. The normalised heavy REE values of the Neoproterozoic granitic gneisses are flat and generally higher than those of the LWZ Fm. The Cretaceous syenite features large variations in its normalised values, which are obviously higher than those of the other two rock types, and the light REEs exhibit poor differentiation (figure 10c).

The REE differences between the LWZ Fm, the Neoproterozoic granitic gneiss and the Lower Cretaceous syenite in the North American Shale 

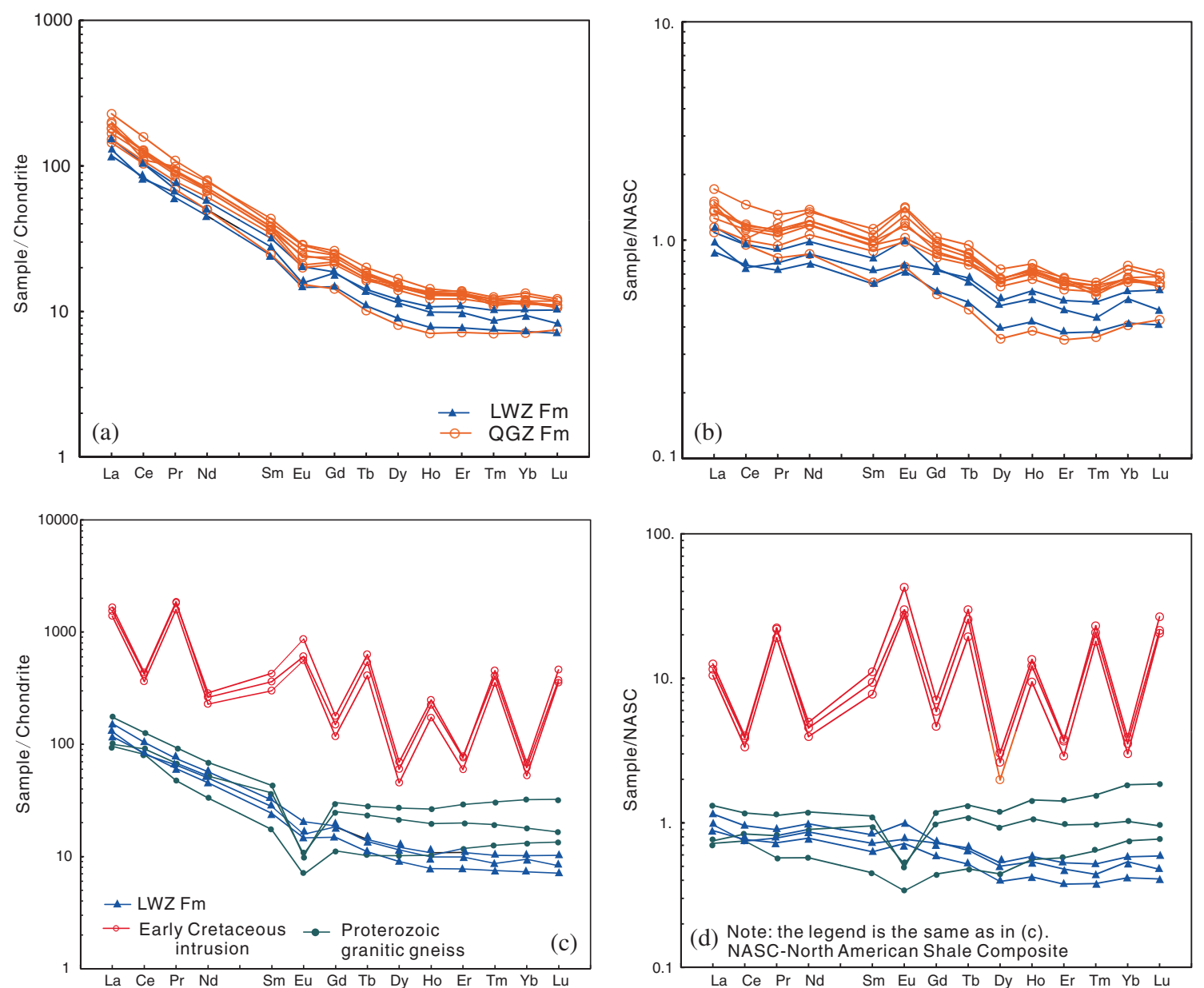

Figure 10. Comparison of the REE-normalized distribution pattern diagrams of the Laiyang Group's sandstones with the Proterozoic and Mesozoic intrusive rocks. (a) Chondrite normalization of the Zhucheng Basin sediments; (b) North American Shale Composite normalization of the Zhucheng Basin sediments; (c) chondrite-normalized comparison of the Proterozoic and Cretaceous intrusive rocks and the LWZ Fm from the Zhucheng Basin; and (d) North American Shale Compositenormalized comparison of the Proterozoic and Cretaceous intrusive rocks and the LWZ Fm from the Zhucheng Basin. The standardized data are from Sun and McDonough (1989).

Composite-normalised diagram are similar to those in the chondrite-normalised diagram (figure 10d). In particular, the Neoproterozoic granitic gneisses feature a negative Eu anomaly.

The above comparison shows that the Laiyang Group sandstones have a substantial affinity to the Jiaonan Orogen's Neoproterozoic granitic gneiss and that the sandstones are unrelated to the Mesozoic intrusive rocks. These findings are consistent with the zircon geochronology of the Laiyang Group's conglomerates.

\section{The tectonic setting and provenance}

\subsection{Tectonic setting}

Under supergene conditions, the migration of major and trace elements in sediments is controlled by various factors, of which the tectonic setting and provenance are the most important (Bhatia 1983).
For clastic sedimentary rocks, the stability of the provenance's tectonic setting controls the derivation of sediments and determines the relative stay duration of major and trace elements at the earth's surface due to weathering denudation, consolidation diagenesis, and then controls the fractionation of major and trace elements. These processes produce different geochemical features in sedimentary rocks (Adeigbe and Jimoh 2013). Hence, the analysis and interpretation of the geochemical features of clastic rocks is an effective way to determine the tectonic setting and provenance of sediments (Ejeh et al. 2013; Ikhane et al. 2014).

The major element $\mathrm{K}_{2} \mathrm{O} / \mathrm{Na}_{2} \mathrm{O}-\mathrm{w}\left(\mathrm{SiO}_{2}\right) \%$ discrimination diagram is based on the correlation of $\mathrm{SiO}_{2}$ and $\mathrm{K}_{2} \mathrm{O} / \mathrm{Na}_{2} \mathrm{O}$ and is used to discriminate between several tectonic settings. This type of diagram does not list continental island arcs due to the similarities in the $\mathrm{SiO}_{2}, \mathrm{~K}_{2} \mathrm{O}$ and $\mathrm{Na}_{2} \mathrm{O}$ signatures of inactive continental margins and continental 

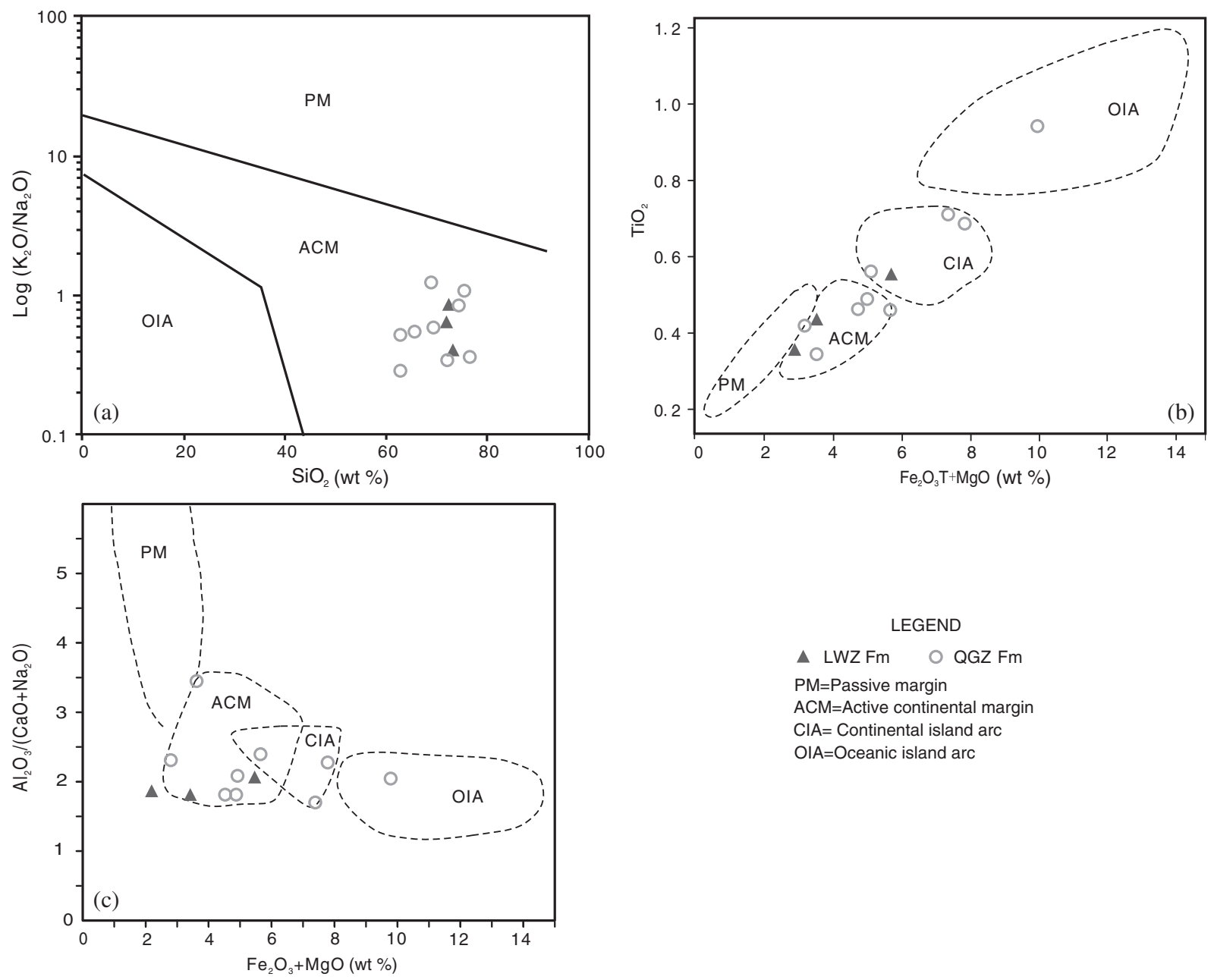

Figure 11. Tectonic discrimination diagram of the major elements in the Laiyang Group's sandstones from the Zhucheng Basin. (a) $\mathrm{K}_{2} \mathrm{O} / \mathrm{Na}_{2} \mathrm{O}-\mathrm{w}\left(\mathrm{SiO}_{2}\right)$ discrimination diagram (Roster and Korsch 1986); (b) $\mathrm{F}_{1}-\mathrm{F}_{2}$ discrimination diagram for major elements (Roster and Korsch 1988); (c) $\mathrm{Fe}_{2} \mathrm{O}_{3}+\mathrm{MgO}-\mathrm{TiO}_{2} \%$ discrimination diagram $(\mathrm{Bhatia} 1983) ;\left(\right.$ d) $\mathrm{Fe}_{2} \mathrm{O}_{3}+$ $\mathrm{MgO}-\mathrm{Al}_{2} \mathrm{O}_{3} /\left(\mathrm{CaO}+\mathrm{Na}_{2} \mathrm{O}\right)$ discrimination diagram (Bhatia 1983).

island arcs (Roster and Korsch 1988). According to this diagram, the plotted points of the sandstones from the LWZ Fm and QGZ Fm fall in the active continental margin area, far from the passive continental margin and ocean island arc areas (figure 11a).

Based on the $\mathrm{Fe}_{2} \mathrm{O}_{3}+\mathrm{MgO}-\mathrm{w}\left(\mathrm{TiO}_{2}\right) \%$ and $\mathrm{Fe}_{2} \mathrm{O}_{3}+\mathrm{MgO}-\mathrm{Al}_{2} \mathrm{O}_{3} /\left(\mathrm{CaO}+\mathrm{Na}_{2} \mathrm{O}\right)$ discrimination diagrams (Bhatia 1983) (figure 11b, c), the majority of the sample points for the LWZ Fm and QGZ Fm lie in the active continental margin and continental island arc provenance fields. The sedimentary sequence from the LWZ Fm to the QGZ Fm transitions from the active continental margin field to the continental island arc field (figure 11b, c). The major element oxide contents of the rocks in the LWZ Fm and QGZ Fm also exhibit a similar trend with respect to the values of active continental margins and continental island arcs (table 1).

In addition to major elements, trace elements are also used to discriminate tectonic settings, such as in the $\mathrm{Th}-\mathrm{Sc}-\mathrm{Zr}$ /10 diagram and the La-Th-Sc diagram (Bhatia and Crook 1986). These diagrams can clearly discriminate between the tectonic settings of ocean island arcs, continental island arcs, active continental margins and passive continental margins. According to these diagrams, the Laiyang Group's sandstones in the Zhucheng Basin primarily plot within the continental island arc field, although a few points fall outside this area (figure 12).

Despite the difference in the results, the discussed diagrams generally indicate that the source rocks of the sediments in the Laiyang group were formed in an active continental margin and/or continental arc setting. Uplifted basement is known to act as a denudation area of active continental margins, and incised magmatic arc is the source areas of continental island arcs (Bhatia 1983). The observed provenance properties indicate that the source areas of the sediment in the Zhucheng Basin experienced intense uplift during the deposition of 
the Laiyang Group and that the magmatic arc, which was originally deeply buried in the basement, began to experience denudation. These results are consistent with the results obtained from the previously discussed sandstone chemical alteration indices and source area paleo-weathering estimates in this paper.

\subsection{Provenance}

It is important to discover the provenance of the Laiyang Group's sandstones in the Zhucheng Basin. The paleocurrent directions indicate the provenance should be in the south-east direction of the basin. The zircon geochronologic data from the conglomerates at the base of the Laiyang Group indicate that the rocks exposed in the source area during the early stages of basin formation were primarily Neoproterozoic granitic gneiss (table 3). The ages of the Neoproterozoic granitic gneisses that crop out in the Jiaonan Orogen are between 739 and $819 \mathrm{Ma}$ (Hacker et al. 2006; Huang et al. 2006). The age of the granitic gneisses in conglomerates is close to that of the Neoproterozoic granitic gneiss in the Jiaonan Orogen. Combined with the paleocurrent directions and the age of the granitic gneiss in conglomerates, the Jiaonan Orogen was the most important provenance of sediment in the Laiyang Group in the Zhucheng Basin. This conclusion is supported by the similarities in both the major element and trace element concentrations between the Laiyang Group's sandstones and the
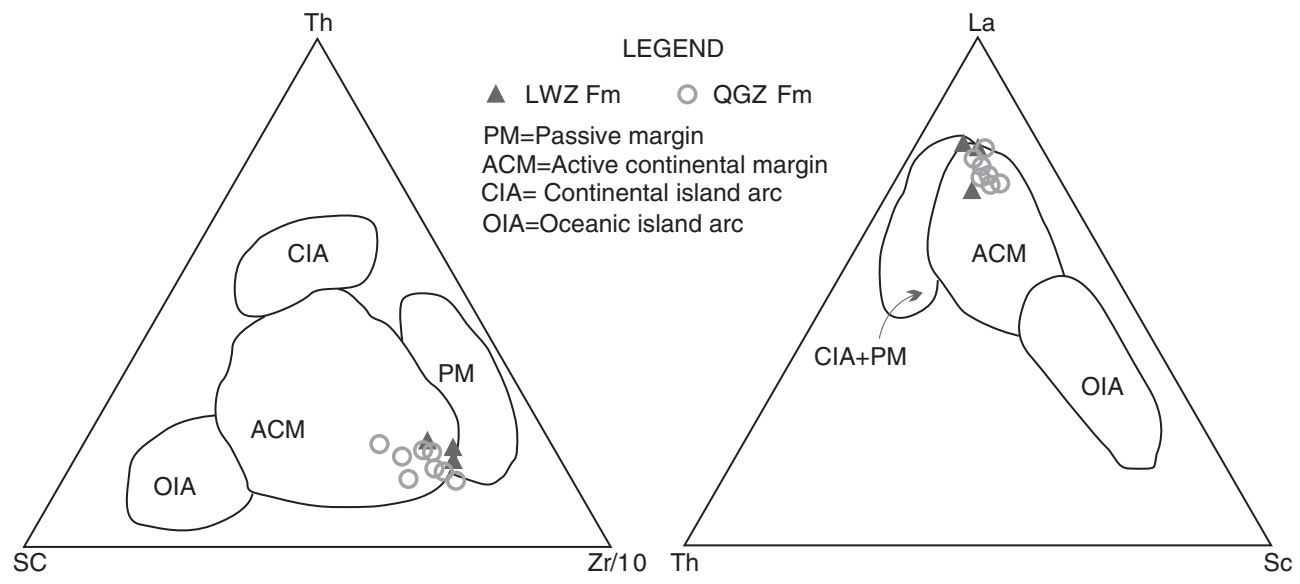

Figure 12. Trace element discrimination diagram for the Laiyang Group's sandstones from the Zhucheng Basin.

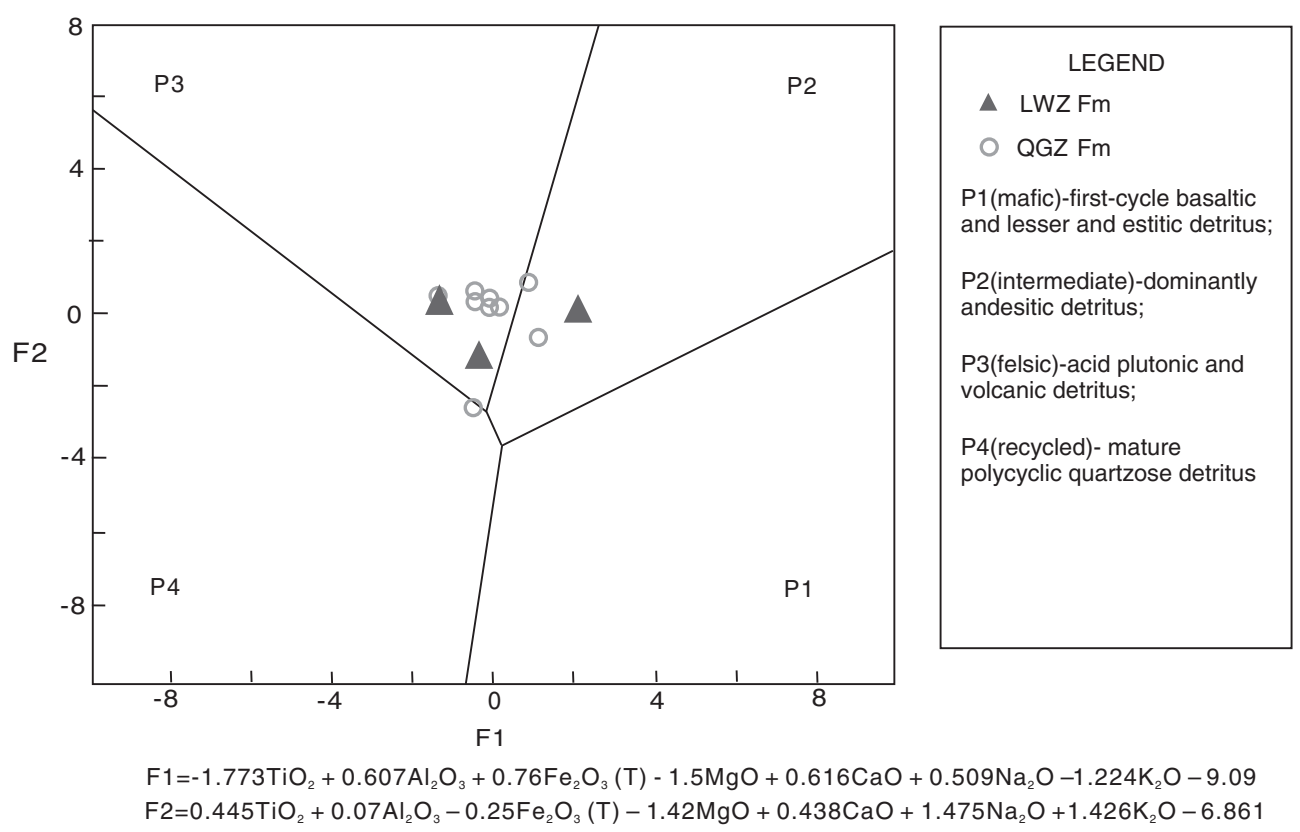

Figure 13. Multivariable function and discrimination diagram of the provenance properties of the major elements of the Laiyang Group's sandstones from the Zhucheng Basin. 
Jiaonan Orogen's Neoproterozoic granitic gneiss. It must be mentioned that, since we found the zircon with the age of $882 \pm 26 \mathrm{Ma}$, there might be other source rocks.

The younger intrusions in the Jiaonan Orogen formed primarily in the Early Cretaceous $(\sim 126$ and $116 \mathrm{Ma}$ ) (Yang et al. 2005; Hacker et al. 2009) (table 4) and featured widely different trace element patterns relative to the Laiyang Group. These results suggest that the Cretaceous intrusive rock was emplaced while the basin was subsiding and was not exposed at the surface.

In addition to geochronologic differences, the established $\mathrm{F}_{1}-\mathrm{F}_{2}$ discrimination function diagram, based on the oxides of $\mathrm{Ti}, \mathrm{Al}, \mathrm{Mg}, \mathrm{Ca}, \mathrm{Na}$ and $\mathrm{K}$, is commonly used to effectively discriminate between mafic, intermediate and felsic magmatic rocks and quartz sedimentary rocks (figure 13) (Roster and Korsch 1988; Ejeh et al. 2013; Zhang et al. 2014).
We analysed the correlations between the Laiyang Group's sandstone samples from the Zhucheng Basin, established a discrimination equation and plotted the points (figure 13). The results show that most of the sample points fall into the felsic igneous rock provenance field, and a few sample points fall into the intermediate rock provenance field. Although the sandstone samples are divided into two provenances, the sample points are tightly grouped near the provenance field of intermediate rocks, indicating that the provenance of the Laiyang Group in the Zhucheng Basin is primarily intermediate-acid igneous rocks. This finding is consistent with the conclusion that the sandstones are primarily ferruginous sandstones and clastic sandstones (according to the geochemical classification diagram for the sandstone's major elements; figure 7) and contain abundant plagioclase (according to the $\mathrm{A}-\mathrm{CN}-\mathrm{K}$ diagram; figure 8).

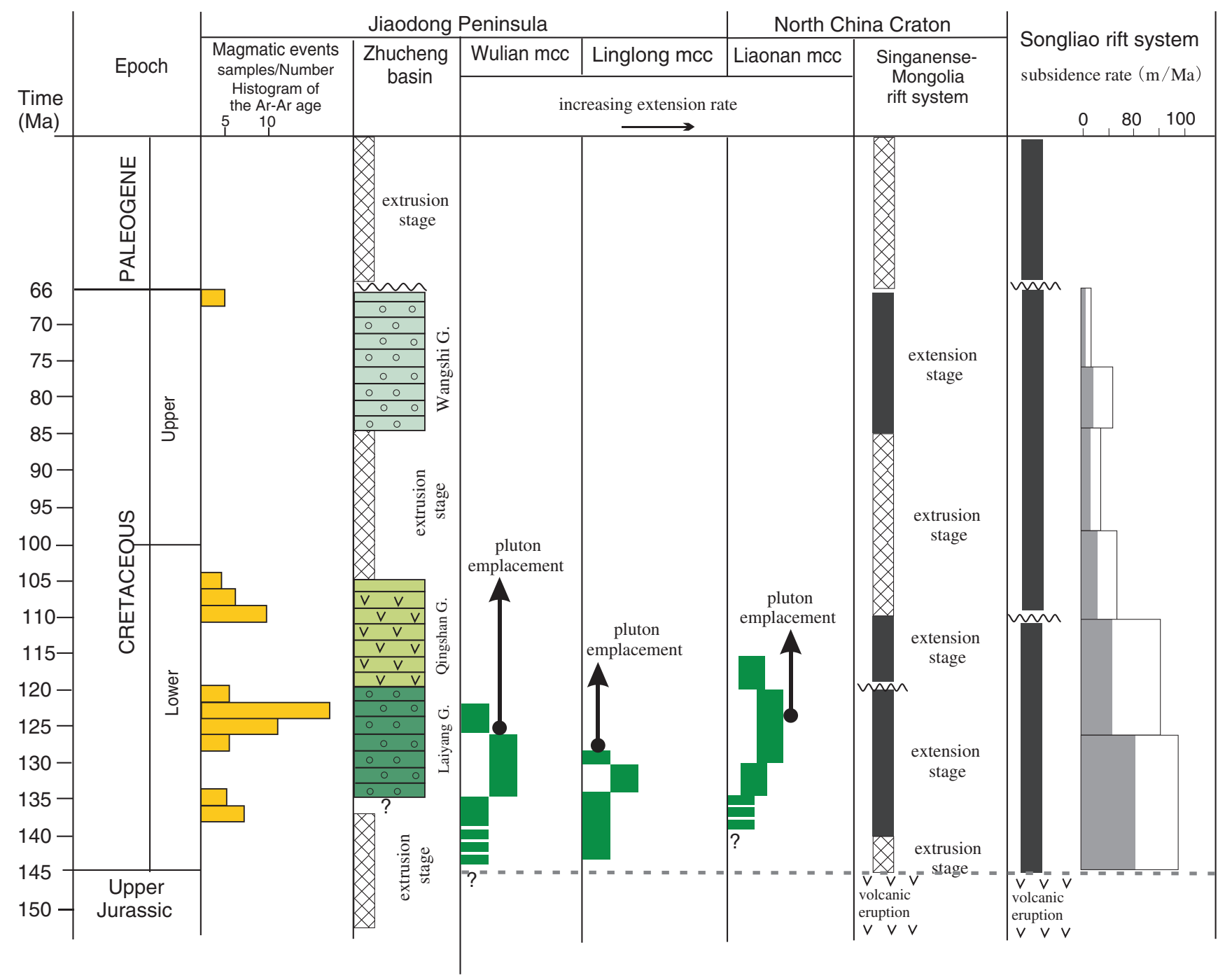

Figure 14. Regional Mesozoic depositional, tectonic and magmatic events in east China. The Ar-Ar ages are from Zhao et al. (1998), Yang et al. (2005), Webb et al. (2006), Hacker et al. (2009), and Guo et al. (2013). The depositional stage of the Zhucheng Basin is from Zhang et al. (2008), the extensional rate of the Liaonan MCC is from Liu et al. (2005), the extensional rate of the Jiaobei Block is from Charles et al. (2011a, 2013), and the subsidence rate is from Ren et al. (2002). 
The rocks in the Jiaonan Orogen are mainly composed of ultrahigh-pressure metamorphic rocks from the Paleoproterozoic Fenzishan Group and Neoproterozoic granitic gneiss and granitic gneiss (Ni et al. 2013). Granitic gneiss is the largest component of the ultrahigh-pressure metamorphic complex, accounting for $75 \%$ of the entire complex's outcropping area (Liu et al. 2008; Zheng et al. 2010). This observation agrees with the results obtained from the geochemical discrimination diagram, indicating that the sandstone's provenance includes intermediate rocks and felsic igneous rocks. The Fenzishan Group and granitic gneiss were the source rocks, which is consistent with the conclusion that the sediments' provenances included active continental margins and continental island arcs.

\section{Tectonic implications}

The geochemical features and provenance analysis of the major elements of the Laiyang Group's sandstones from the Zhucheng Basin indicate that the southern Sulu Orogen (the Jiaonan Orogen) experienced rapid uplift during rapid subsidence of the Zhucheng Basin at 135-125 Ma (the depositional period of the Laiyang Group) (Zhang et al. 2003). These events occurred approximately 60$80 \mathrm{Ma}$ later than the ultrahigh-pressure metamorphism and continental collision events, which is longer than the generally accepted interval between continental collision and crustal remelting events (Thompson 1999). Therefore, the rapid uplift of this orogen is likely unrelated to the post-orogenic extension and is instead associated with a separate extensional event. A series of extensional features have been described in the northern Jiaodong area and include the formation and denudation of the Linglong MCC (130-124 Ma) (Charles et al. 2011a) and the uplift and denudation of the Jiaobei Uplift (140-110 Ma) (Zhang et al. 2006). Additionally, a series of metamorphic core complexes and detachment fault zones, including the Liaonan MCC (Liu et al. 2005, 2006, 2011) and the Waziyu MCC (Li et al. 2012) (figure 1), was developed on the opposite side of the Jiaodong peninsula. In fact, the development phase of the Laiyang Group in the Zhucheng Basin coincides with the intense destruction of the North China Craton (Liu et al. 2005; Zhu et al. 2011; Ni et al. 2014) (figure 14). During this period, widespread rifted basins developed in the North China Craton, resulting in the Xing'anling-Monglolia giant rift system (Meng 2003a; Meng et al. 2003b; Ni et al. 2011), Songliao rift system (Song et al. 2010; Zhang et al. 2011), Lower Cretaceous Bohai Bay rift system (Ren et al. 2002), and others (figure 1).

The rapid subsidence of the Zhucheng Basin during the formation of the Laiyang Group and the rapid uplift of the southern Sulu Orogen are both the result of a single regional extensional event. During this event, rifted basins formed in the shallow crust, and the Wulian MCC developed in and was exhumed from the deeper crust. These tectonic phenomena are the result of the lithospheric thinning of the North China Craton and its peripheral areas.

\section{Conclusions}

- The fluvial-lacustrine facies sedimentary rocks in the Laiyang Group and the volcanic rocks in the Qingshang Group formed during the Early Cretaceous in the Zhucheng Basin. The Linshansi Formation's conglomerates and the LWZ Fm, QGZ Fm and Fajiaying Formation's sandstones and mudstones were successively deposited, forming the Laiyang Group.

- The major element contents of the LWZ Fm and QGZ Fm in the Zhucheng Basin are similar, but differences still exist. The LWZ Fm has higher $\mathrm{SiO}_{2}$ contents (average: 72.87 wt.\%) than the QGZ Fm (average: 70.26 wt.\%), and the other major element contents, apart from $\mathrm{CaO}$, are lower in the former than in the latter. The values of the LWZ Fm (lower Laiyang Group) in various indices are similar to the average values of active continental margins, whereas the values of the QGZ Fm (upper Laiyang Group) are similar to those of continental island arcs.

- The majority of the sandstone samples from the Laiyang Group in the Zhucheng Basin are ferruginous sandstones and greywackes, although a few samples are lithic sandstones. Therefore, the sandstones did not experience intense weathering and were deposited near the source region or experienced rapid diagenesis. In addition, mafic material was abundant in the source areas. The tectonic setting discrimination diagram reveals that the provenance of the Laiyang Group includes features of active continental margins and continental island arcs.

- The trace element and REE contents of the LWZ Fm and QGZ Fm in the Zhucheng Basin are similar. In the primitive mantle-normalised trace element spider diagram, the Laiyang Group is relatively enriched in the large ion lithophile elements Rb, Ba and $\mathrm{K}$ and REEs La and $\mathrm{Ce}$ and is depleted in the inactive elements $\mathrm{P}, \mathrm{Ti}$, $\mathrm{Y}, \mathrm{Yb}$ and $\mathrm{Lu}$, with intermediate amounts of $\mathrm{Zr}$ and $\mathrm{Hf}$ and various $\mathrm{Sr}$ contents. In the chondritenormalised diagram, the Laiyang Group is relatively enriched in the light REEs and relatively depleted in the heavy REEs, with negative Eu anomalies. The trace element and REE characteristics of the Laiyang Group are similar to 
those of the Neoproterozoic granitic gneisses in the Jiaonan Orogen but are significantly different from those of the Lower Cretaceous intrusions.

- The zircon age of the granitic gneiss material in the conglomerates at the base of the Laiyang Group is $790 \pm 8.4 \mathrm{Ma}$, close to the ages of the Neoproterozoic granitic gneisses in the Jiaonan Orogen (739-819 Ma). Clearly, these ages differ significantly from those of the Lower Cretaceous intrusions, revealing that the Neoproterozoic granitic gneisses in the Jiaonan Orogen may be the main provenance of the Laiyang Group in the Zhucheng Basin.

- The chemical alteration and paleo-weathering indices (CIA, CIW, PIA and ICV) suggest that the source rocks generally did not experience weathering during the depositional period of the Laiyang Group. The Jiaonan Orogen experienced rapid uplift during the depositional period of the Laiyang Group in conjunction with the high exhumed rate of $2.0 \mathrm{~km} \mathrm{Ma}^{-1}$ from before $c .128$ Ma to $123 \mathrm{Ma}$ in Jiaonan Orogen.

- The rapid subsidence of the Zhucheng Basin during the formation of the Laiyang Group and the rapid uplift of the Jiaonan Orogen both resulted from a single regional extensional event. Rifted basins and the Wulian MCC also formed during this event. These tectonic phenomena represent significant shallow structural responses to the lithospheric thinning of the North China Craton and its peripheral areas.

\section{Acknowledgements}

This study was supported by the National Natural Science Foundation of China (No. 91214301, No. 41172089, No. 41402250 and No. 41430211), the Natural Science Foundation of Shandong Province (No. ZR2013DM007) and the Science Foundation of Key Laboratory of Gold Mineralization Processes and Resource Utilization subordinated to the Ministry of Land and Resources (No. 2013006).

\section{References}

Adeigbe O C and Jimoh Y A 2013 Geochemical fingerprints: Implication for provenance, tectonic and depositional settings of Lower Benue Trough Sequence, southeastern Nigeria; J. Env. Earth Sci. 3 115-140.

Andersen T 2002 Correction of common lead in U-Pb analyses that do not report Pb-204; Chem. Geol. 192 59-79.

Bhatia M R 1983 Plate tectonics and geochemical composition of sandstones; J. Geol. 91 611-627.

Bhatia M R and Crook K A 1986 Trace-element characteristics of greywackes and mudrocks: Provenance and tectonic control; Contrib. Mineral. Petrol. 92 181-193.
Billia M A, Timms N E, Toy V G, Hart R D and Prior D J 2013 Early Cretaceous Provenance change in the southern Hailar Basin, northeastern China and its implication for basin evolution; Cretaceous Res. 40 21-42.

Charles N, Gumiaux C, Augier R, Chen Y, Zhu R X and Lin W 2011a Metamorphic core complexes vs. synkinematic plutons in continental extension setting: Insights from key structures (Shandong Province, eastern China); J. Asian Earth Sci. 40 261-278.

Charles N, Chen Y, Augier R, Gumiaux C, Lin W, Faure $\mathrm{M}$, Monié $\mathrm{P}$, Choulet $\mathrm{F}$, Wu F, Zhu $\mathrm{R}$ and Wang Q 2011b Palaeomagnetic constraints from granodioritic plutons (Jiaodong peninsula): New insights on Late Mesozoic continental extension in eastern Asia; Phys. Earth Planet. Inter. 187 276-291.

Charles N, Augier R, Gumiaux C, Monié P, Chen Y, Faure $\mathrm{M}$ and Zhu R 2013 Timing, duration and role of magmatism in wide rift systems: Insights from the Jiaodong peninsula (China, east Asia); Gondwana Res. 24 412-428.

Chen R, Zheng Y and Xie L 2010 Metamorphic growth and recrystallization of zircon: Distinction by simultaneous in-situ analyses of trace elements, $\mathrm{U}-\mathrm{Th}-\mathrm{Pb}$ and $\mathrm{Lu}-\mathrm{Hf}$ isotopes in zircons from eclogite-facies rocks in the Sulu orogen; Lithos 114 132-154.

Cox R and Lowe D R 1995 A conceptural review of regionalscale controls on the composition of clastic sediment and the co-evolution of continental blocks and their sedimentary cover; J. Sedim. Res. 65 1-12.

Cox R, Lowe D R and Cullers R L 1995 The influence of sediment recycling and basement composition on evolution of mudrock chemistry in the southwestern United States; Geochim. Cosmochim. Acta 59 2919-2940.

Ejeh O I, Etobro A A I and Adaikpoh E O 2013 Pattern of geochemical and sedimentological variability of the Albian to Cenomanian Upper Bima Sandstone, Benue Trough, Nigeria: Implications on tectonic provenance and source area weathering; J. Env. Earth Sci. 3 170-184.

Erikson J P, Kelley S A, Osmolovsky P and Verosub K L 2012 Linked basin sedimentation and orogenic uplift: The Neogene Barinas basin sediments derived from the Venezuelan Andes; J. S. Am. Earth Sci. 39 138-156.

Fedo C M, Nesbitt H W and Young G M 1995 Unraveling the effects of potassium metasomatism in sedimentary rocks and paleosols, with implications for paleoweathering conditions and provenance; Geology 23 921-924.

Gao S, Zhang B R, Gu X M, Xie Q L, Gao C L and Guo X M 1995 Silurian-Devonian provenance changes of south Qinling basins: Implications for accretion of the Yangtze (South China) to the North China Cratons; Tectonophys. 250 183-197.

Goss S C, Wilde S A, Wu F Y and Yang J H 2010 The age, isotopic signature and significance of the youngest Mesozoic granitoids in the Jiaodong Terrane, Shangdong Province, North China Craton; Lithos 120 309-326.

Grimmer J C, Ratschbacher L, McWilliams M, Franz L, Gaitzsch I, Tichomirowa M, Hacker B R and Zhang Y 2003 When did the ultrahigh-pressure rocks reach the surface? A ${ }^{207} \mathrm{~Pb} /{ }^{206} \mathrm{~Pb}$ zircon, ${ }^{40} \mathrm{Ar} /{ }^{39} \mathrm{Ar}$ white mica, Si-in-white mica, single-grain provenance study of Dabie Shan synorogenic foreland sediments; Chem. Geol. 197 87-110.

Guo J H, Chen F K, Zhang X M, Siebel W and Zhai M G 2005 Evolution of syn- to post-collisional magmatism from north Sulu UHP belt, eastern China: Zircon U-Pb geochronology; Acta Petrol. Sin. 21 1281-1301.

Guo P, Santosh M and Li S 2013 Geodynamics of gold metallogeny in the Shandong Province, NE China: An integrated geological, geophysical and geochemical perspective; Gondwana Res. 24 1172-1202. 
Hacker B R, Wallis S R, McWilliams M O and Gans P B $2009{ }^{40} \mathrm{Ar} /{ }^{39} \mathrm{Ar}$ Constraints on the tectonic history and architecture of the ultrahigh-pressure Sulu orogen; J. Metamorph. Geol. 27 827-844.

Hacker B R, Wallis S R, Ratschbacher L, Grove M and Gehrels G 2006 High-temperature geochronology constraints on the tectonic history and architecture of the ultrahigh-pressure Dabie-Sulu Orogen; Tectonics 25 $1-17$.

Harnois L 1988 The CIW index: A new chemical index of weathering; Sedim. Geol. 55 319-322.

Herron M M 1988 Geochemical classification of terrigenous sands and shales from core or log data; J. Sedim. Res. 58 820-829.

Holt P J, Allen M B and Hunen J V 2015 Basin formation by thermal subsidence of accretionary orogens; Tectonophys. $639132-143$.

Huang J, Zheng Y and Zhao Z 2006 Melting of subducted continent: Element and isotopic evidence for a genetic relationship between Neoproterozoic and Mesozoic granitoids in the Sulu orogen; Chem. Geol. 229 227-256.

Ikhane P R, Akintola A I, Bankole S I and Oyinboade Y T 2014 Provenance studies of sandstone facies exposed near igbile southwestern Nigeria: Petrographic and geochemical approach; J. Geogr. Geol. 6 47-68.

Jackson S E, Pearson N J, Griffin W L and Belousova E A 2004 The application of laser ablation-inductively coupled plasma-mass spectrometry to in situ $\mathrm{U}-\mathrm{Pb}$ zircon geochronology; Chem. Geol. 211 47-69.

Jason R P and Michale A V 2003 Chemical weathering indices applied to weathering profiles developed on heterogeneous felsic metamorphic parent rocks; Chem. Geol. $202397-416$.

Li C, Wang J, Zhou Z, Geng J, Chen B, Yang F, Wu J, Yu P, Zhang X and Zhang S 2012 3D geophysical characterization of the Sulu-Dabie orogen and its environs; Phys. Earth Planet. Inter. 192-193 35-53.

Li G, Liu Z, Li S, Xu Z, Dong X and Liu J 2012 Crustal thinning during Mesozoic extensional detachment faulting in the Yiwulüshan region, eastern North China Craton; Int. Geol. Rev. 55 749-766.

Li S, Huang G and Li H 2002 Post-collisional lithosphere delamination of the Dabie-Sulu orogen; Chinese Sci. Bull. 47 259-263.

Li S Y, Meng Q R, Li R W, Wang D X and Chu S W 2008 Characteristics of material components from the Lower Cretaceous Laiyang Formation in Jiaolai basin, Shangdong province, eastern China and constraints to the provenance; Acta Petrol. Sin. 24 2395-2406.

Li S Z, Liu X, Suo Y H, Liu L P, Qian C C, Liu X C, Zhang G W and Zhao G C 2009 Triassic folding and thrusting in the Eastern Block of the North China Craton and the Dabie-Sulu orogen and its geodynamics; Acta Petrol. Sin. 25 2031-2049.

Liu F, Gerdes A, Zeng L and Xue H 2008 SHRIMP U-Pb dating, trace elements and the $\mathrm{Lu}-\mathrm{Hf}$ isotope system of coesite-bearing zircon from amphibolite in the SW Sulu UHP terrane, eastern China; Geochim. Cosmochim. Acta 72 2973-3000.

Liu J L, Davis G A, Lin Z Y and Wu F Y 2005 The Liaonan metamorphic core complex, southeastern Liaoning Province, North China: A likely contributor to Cretaceous rotation of eastern Liaoning, Korea and contiguous areas; Tectonophys. 407 65-80.

Liu J L, Guan H M, Ji M, Cao S Y and Hu L 2006 The Liaonan metamorphic core complex: Constitution, structure and evolution; Acta Geol. Sin. 80 502-513.

Liu J L, Ji M, Shen L, Guan H M and Davis G A 2011 Early Cretaceous extensional structures in the Liaodong peninsula: Structural associations, geochronological constraints and regional tectonic implications; Sci. China Ser. D 54 823-842.

Liu J L, Shen L, Ji M, Guan H M, Zhang Z C and Zhao Z D 2013 The Liaonan/Wanfu metamorphic core complexes in the Liaodong peninsula: Two stages of exhumation and constraints on the destruction of the North China Craton; Tectonics 32 1121-141.

Luo J H, Che Z C, Zhang G F, Nian X Q and Zhang X L 2012 Early-Middle Permian basin-mountain coupling features between northwestern margin of the Tarim basin and the south Tianshan orogen; Acta Petrol. Sin. 28 2506-2514.

Ma L, Jiang S, Hou M, Dai B, Jiang Y, Yang T, Zhao K, Pu W, Zhu Z and Xu B 2014 Geochemistry of Early Cretaceous calc-alkaline lamprophyres in the Jiaodong peninsula: Implication for lithospheric evolution of the eastern North China Craton; Gondwana Res. 25 859-872.

McLennan S M 1993 Weathering and global denudation; J. Geol. 101 295-303.

McLennan S M, Hemming S, McDaniel D K and Hanson G N 1993 Geochemical approaches to sedimentation, provenance, and tectonics; Geol. Soc. Am. Spec. Paper 284 21-40.

Meng Q 2003a What drove late Mesozoic extension of the northern China-Mongolia tract? Tectonophys. 369 $155-174$.

Meng Q R, Hu J M, Jin J Q, Zhang Y and Xu D F 2003b Tectonics of the late Mesozoic wide extensional basin system in the China-Mongolia border region; Basin Res. 16 397-415.

Meng Q R, Li S Y and Li R W 2007 Mesozoic evolution of the Hefei basin in eastern China: Sedimentary response to deformations in the adjacent Dabieshan and along the Tanlu fault; Geol. Soc. Am. Bull. 119 897-916.

Ni J L, Liu J L, Tang X L, Zhao C Q and Zeng Q D 2014 Evolution of the ductile shear zone of the Paishanlou gold deposits, western Liaoning, China; Sci. China Ser. D $5 \mathbf{7}$ 600-613.

Ni J L, Guo Y, Wang Z M, Liu J L, Lin Y X and Li Y 2011 Tectonics and mechanisms of uplift in the central uplift belt of the huimin depression; J. Earth Sci. 22 299-315.

Ni J L, Liu J L, Tang X L, Yang H B, Xia Z M and Guo Q J 2013 The Wulian metamorphic core complex: A newly discovered metamorphic core complex along the Sulu Orogenic Belt, eastern China; J. Earth Sci. 24 1-22.

Ni J L, Liu J L, Tang X L, Yang H B, Xia Z M and Zhang T T 2016 Early Cretaceous exhumation of the Sulu orogenic belt as a consequence of the eastern Eurasian tectonic extension: Insights from the newly discovered Wulian metamorphic core complex, eastern China; J. Geol. Soc. London, doi: 10.1144/jgs2014-122.

Pettijohn F J, Potter P E and Siever R 1972 Sand and Sandstone; Springer-Verlag, New York, 618p.

Ren J Y, Tamaki K, Li S T and Zhang Z X 2002 Late Mesozoic and Cenozoic rifting and its dynamic setting in Eastern China and adjacent areas; Tectonophys. 344 175-205.

Roster B P and Korsch R J 1988 Provenance signatures of sandstone--mudstone suites determined using discriminant function analysis of major-element data; Chem. Geol. 67 119-139.

Rudnick R L and Gao S 2003 Composition of the continental crust; In: Treatise on Geochemistry (eds) Holland H D and Turekian K K, Elsevier-Pergamon, Oxford, pp. 1-64.

Song L Z, Zhao Z H, Jiao G H, Sun P, Luo X, Jiang X H, Wang Z H, Zeng F Y and Miao W D 2010 Geochemical characteristics of Early Cretaceous volcanic rocks from Songliao basin, northeast China, and its tectonic implications; Acta Petrol. Sin. 26 1182-1194. 
Sun S S and McDonough W F 1989 Chemical and isotopic systematics of oceanic basalts: Implications for mantle composition and processes; In: Magmatism in the Ocean Basins (eds) Saunders A D and Norry M J, Geol. Soc. London Spec. Publ. 42 313-345.

Suo S, Zhong Z, Zhou H, You Z and Zhang L 2012 Two fresh types of eclogites in the Dabie-Sulu UHP metamorphic belt, China: Implications for the deep subduction and earliest stages of exhumation of the continental crust; J. Earth Sci. 23 775-785.

Tang H, Zheng J, Yu C, Ping X and Ren H 2014 Multistage crust-mantle interactions during the destruction of the North China Craton: Age and composition of the Early Cretaceous intrusions in the Jiaodong peninsula; Lithos 190-191 52-70.

Thompson A B 1999 Some time-space relationships for crustal melting and granitic intrusion at various depths; In: Understanding granites: Integrating new and classical techniques (ed) Castro A; Geol. Soc. London Spec. Publ. $1687-25$.

Wang L, Kusky T M, Polat A, Wang S, Jiang X, Zong K, Wang J, Deng H and Fu J 2014 Partial melting of deeply subducted eclogite from the Sulu orogen in China; Nature Communications 5 5604-5615.

Wang L, Kusky T M and Santosh M 2012 On the role of dual active margin collision for exhuming the world's largest ultrahigh pressure metamorphic belt; J. Earth Sci. 23 802-812.

Wang Q C, Li R W, Wang D X and Li S Y 2003 Eclogites preserved as pebbles in Jurassic conglomerate, Dabie Mountains, China; Lithos $\mathbf{7 0}$ 345-357.

Webb L E, Graham S A, Johnson C L, Badarch G and Hendrix M S 1999 Occurrence, age, and implication of the Yagan-Onch Hayrhan metamorphic core complex, southern Mongolia; Geology 27 143-146.

Webb L E, Ratschbacher L, Hacker B R and Dong S W 2001 Kinematics of exhumation of high- and ultrahigh-pressure rocks in the Hong'an and Tongbai Shan of the QinlingDabie collisional orogen, eastern China; Geol. Soc. Am. Memoir 194 413-434.

Webb L E, Leech M L and Yang T N $2006{ }^{40} \mathrm{Ar} /{ }^{39} \mathrm{Ar}$ thermochronology of the Sulu terrane: Late Triassic exhumation of high- and ultrahigh-pressure rocks and implications for Mesozoic tectonics in East Asia; In: Ultrahigh-pressure metamorphism: Deep continental subduction (eds) Hacker B R, McClelland W C and Liou J G, Geol. Soc Am. Spec. Paper, pp. 77-92.

Wiedenbeck M, Alle P, Corfu F, Griffin W L, Meier M, Oberku F, Vonquadt A, Roddick J C and Speigel W 1995 Natural zircon standards for U-Th-Pb, Lu-Hf, traceelement and Ree analyses; Geostandards NewsLetter 19 $1-23$.

Wu M, Zhao G, Sun M, Li S, Bao Z, Tam P Y, Eizenh Efer P R and He Y 2014 Zircon U-Pb geochronology and Hf isotopes of major lithologies from the Jiaodong Terrane: Implications for the crustal evolution of the eastern block of the North China Craton; Lithos 190 71-84.

Xiao W J, Windley B F, Hao J and Zhai M G 2003 Accretion leading to collision and the Permian Solonker suture,
Inner Mongolia, China: Termination of the central Asian orogenic belt; Tectonics 22 1069, doi: 10.1029/2002TC001484.

Xie Y X, Wenk H R and Matthies S 2003 Plagioclase preferred orientation by TOF neutron diffraction and SEM-EBSD; Tectonophys. 370 269-286.

Xie S, Wang $\mathrm{S}$ and Xie H 2015 SHRIMP U-Pb dating of detrital zircons from the Fenzishan Group in eastern Shandong, North China Craton; Acta Petrol. Sin. 30 2989-2998.

Xu H J, Ma C Q and Ye K 2007 Early Cretaceous granitoids and their implications for collapse of the Dabie orogen, eastern China: SHRIMP zircon $\mathrm{U}-\mathrm{Pb}$ dating and geochemistry; Chem. Geol. 240 238-259.

Xu Z Q, Yang W C, Ji S C, Zhang Z M, Yang J S, Wang Q and Tang Z M 2009 Deep root of a continentcontinent collision belt: Evidence from the Chinese Continental Scientific Drilling (CCSD) deep borehole in the Sulu ultrahigh-pressure (HP-UHP) metamorphic terrane, China; Tectonophys. 475 204-219.

Yang J, Wu F, Chung S, Wilde S, Chu M, Lo C and Song B 2005 Petrogenesis of Early Cretaceous intrusions in the Sulu ultrahigh-pressure orogenic belt, east China and their relationship to lithospheric thinning; Chem. Geol. 222 200-231.

Zhang $\mathrm{F}$ Q, Chen $\mathrm{H} \mathrm{L}, \mathrm{Yu} \mathrm{X}$, Dong $\mathrm{C}$ W, Yang $\mathrm{S} \mathrm{F}$, Pang Y M and Batt G E 2011 Early Cretaceous volcanism in the northern Songliao Basin, NE China, and its geodynamic implication; Gondwana Res. 19 163-176.

Zhang H 2012 Destruction of ancient lower crust through magma underplating beneath Jiaodong peninsula, North China Craton: $\mathrm{U}-\mathrm{Pb}$ and $\mathrm{Hf}$ isotopic evidence from granulite xenoliths; Gondwana Res. 21 281-292.

Zhang H F, Li S R, Zhai M G and Guo J H 2006 Crust uplift and its implications in the Jiaodong peninsula, eastern China; Acta Petrol. Sin. 22 285-295.

Zhang Y, Dong S and Shi W 2003 Cretaceous deformation history of the middle Tan-Lu fault zone in Shandong Province, eastern China; Tectonophys. 363 243-258.

Zhang Y, Pe-Piper G and Piper D J W 2014 Sediment geochemistry as a provenance indicator: Unravelling the cryptic signatures of polycyclic sources, climate change, tectonism and volcanism; Sedimentology 61 383-410.

Zhang Y Q, Li J L, Zhang T, Dong S W and Yuan J Y 2008 Cretaceous to Paleocene tectono-sedimentary evolution of the Jiaolai Basin and the contiguous areas of the Shandong peninsula (North China) and its geodynamic implications; Acta Geol. Sin. 82 1229-1257.

Zhao G, Wang D, Cao Q and Yu L 1998 Thermal evolution and its significance of I-A type granitoid complex; Sci. China Ser. D 41 529-536.

Zheng J, Tang H, Zhao J, Su Y, Yu C, Wei Q, Liu Q and Wu X 2010 Age, trace elements and Hf-isotope composition of zircon in eclogites from the Sulu UHP belt, eastcentral China: Neoproterozoic intrusion and subsequent Paleozoic and Early Mesozoic metamorphism; J. Earth Sci. 21 598-622.

Zhu R, Chen L, Wu F and Liu J 2011 Timing, scale and mechanism of the destruction of the North China Craton; Sci. China Ser. D 54 789-797. 